\title{
Staatswissenschaften und Staatspraxis: ein herausfordernd prekäres Verhältnis
}

\author{
von Horst Dreier, Friedrich Wilhelm Graf und Joachim Jens Hesse
}

\begin{abstract}
Das nie einfache und einer kontinuierlichen Diskussion ausgesetzte Verhältnis von Staatswissenschaften und Staatspraxis findet aufgrund zweier komplementärer Entwicklungen neue Aufmerksamkeit: endogen durch jene Herausforderungen tradierter Staatlichkeit, die sich mit Veränderungen der demographischen, ökonomischen und soziokulturellen Rahmenbedingungen verbinden, sowie exogen durch jene Grenzüberschreitungen, die im Gefolge von Europäisierungs- und Globalisierungsprozessen (samt damit einhergehender Souveränitätsverluste) erkennbar werden. Der nachfolgende Beitrag summiert die theoretischen und empirisch-analytischen Schlussfolgerungen, die die Autoren aus den Beiträgen des vorangehenden ZSE-Themenhefts (3-4/2009) ziehen. Im Ergebnis erweist sich das Verhältnis von Staatswissenschaften und Staatspraxis als gleichermaßen prekär wie herausfordernd. Prekär, weil die heutigen Anforderungen an das deutsche Regierungssystem von einer - trotz stabiler Ausgangssituation - ,gefährdeten Stabilität" sprechen lassen, und herausfordernd, weil die schnellen Veränderungen der Rahmenbedingungen oberste Bundesorgane wie gebietskörperschaftliche Einrichtungen unter einen kontinuierlichen Anpassungs-, in Teilen auch Reformdruck stellen.
\end{abstract}

The ongoing debate on the development of the public sector and according forms and functions of traditional "statehood" has gained renewed attention due to two complementary developments: the endogenous challenge of rapidly changing economic, demographic and socio-cultural framework conditions, and the exogenous pressures due to the ongoing processes of Europeanisation and globalisation. The following paper summarises theoretical and empirico-analytical conclusions drawn from the theme-specific contributions to a special issue of the ZSE (3-4/2009). The relationship between the performance of the public sector and the analytical reflections among lawyers, economists and social scientists is seen as both precarious and challenging: precarious due to a situation described - despite stable pre-conditions - as "endangered stability", and challenging as a result of the considerable pressures to adapt to an ever-changing environment and to seek solutions that demand a closer interaction of academic analyses and practical adjustment.

\section{Einleitung}

Der nachfolgende Beitrag summiert die im Themenheft 3-4/2009 der ZSE vorgelegten Analysen, die unter dem Titel „Staatswissenschaften und Staatspraxis: endogene und exogene Herausforderungen tradierter Staatlichkeit" führende 
Rechts-, Wirtschafts- und Sozialwissenschaftler auf eine gemeinsame Fragestellung verpflichteten. ${ }^{1}$ Dieses Unterfangen steht unter einem zweifachen Vorbehalt: zum einen der notwendigen Konzentration auf einige zusammenfassende Erkenntnisse, die in den jeweiligen Einzelbeiträgen dann um vieles ausführlicher diskutiert werden, und zum anderen einer ungewöhnlichen Ausgangssituation, die sich mit den spezifischen Denk- und Arbeitsmustern der drei Autoren (die gleichzeitig als Herausgeber der gesondert erscheinenden Buchpublikation fungieren) verbindet. Während die angesprochenen Herausforderungen zunächst unter der eher staatstheoretischen Frage nach der „Wiederkehr des Staates“ diskutiert werden (II), richtet sich die nachfolgende Argumentation aus staatspraktischer Sicht auf aktuelle wie strukturelle Handlungsbedarfe (III); der abschlieBende Verweis auf ideengeschichtliche Entwicklungslinien erinnert an die nur vermeintliche Stabilität des wissenschaftlichen und gesellschaftlichen Staatsverständnisses (IV). Die Autoren eint die Hoffnung, in der Zusammenführung rechts-, wirtschafts- und sozialwissenschaftlicher Erkenntnisse zu einer dem Gegenstand angemessenen Sichtweise beizutragen, um so eine dringlich benötigte Staatsdiskussion fortzuführen, die wiederum einer kontinuierlichen theoretischen wie empirisch-analytischen Rückbindung bedarf.

\section{Staatstheoretische Grundlegungen}

\section{Schwindende Bedeutung des Nationalstaats?}

Der Staat ist schon des Öfteren totgesagt worden. Als besonders prominent darf einmal mehr die Stimme Carl Schmitts gelten, der im Vorwort zu seinem „Begriff des Politischen“ 1963 dekretierte, die Epoche der Staatlichkeit gehe zu Ende; darüber sei kein weiteres Wort mehr zu verlieren. Ende der Staatlichkeit wurde hier mit steigender Macht der Verbände sowie der politischen Parteien gleichgesetzt und kündete letztlich nur von einer tiefsitzenden Fremdheit gegenüber einer offenen pluralistischen Gesellschaft mit demokratischer Staatsorganisation. Die Abgesänge auf den Staat, die man in den letzten zwei oder drei Jahrzehnten vernehmen konnte, stimmen eine andere Tonart an. Hier geht es nicht um den von Konservativen beklagten, aber in einer demokratischen Gesellschaft geradezu konstitutiven Umstand, dass der Staat eine Art Selbstorganisation der Gesell-

1 Vgl. ZSE 7/3-4 (2009): „Staatswissenschaften und Staatspraxis. Endogene und exogene Herausforderungen tradierter Staatlichkeit“. Eine ergänzte Fassung erscheint im Mai dieses Jahres in einer gesonderten Buchpublikation des Nomos-Verlags. 
schaft ist. Vielmehr erscheint der Staat selbst als fossiles Relikt. Daher konnte er in ambitionierten Theorien einer ganz neuen Welt des Rechts und in entsprechenden Systementwürfen kaum mehr Platz mehr finden. Er schien zu alt, auch zu alteuropäisch, zu starr, zu monolithisch, zu national zu sein, um in der mehr oder minder schönen Welt der Globalisierung mit ihren polyglotten Akteuren und ihren weltumspannenden Handlungsfeldern noch an seine frühere Glanzrolle als Zentralgestirn politischen Handelns und Orientierungspunkt politischer Philosophie wie Staatstheorie anknüpfen zu können. Auf die Frage: Weltstaat oder Staatenwelt? antworteten Vertreter der politischen Philosophie schon einmal mit einem entschieden autosuggestiven: Weltstaat, genauer: Weltrepublik. Und in Politik- und Systemtheorie ist die Rede von Weltgesellschaft, Globalisierung des Rechts, Zerfaserung des Staates oder gleich Abschied von demselben ohnehin seit längerem an der Tagesordnung.

Ganz von ungefähr kam das nicht. Denn es fehlt ja keineswegs an Indizien für einen massiven Relevanzverlust. So schien der Staat zunehmend zerrieben zwischen den schicksalhaft anmutenden Prozessen der Supra- und Internationalisierung auf der einen und Privatisierungsmaßnahmen auf der anderen Seite, und so wurde auch zunehmend unklarer, wo genau er seinen früheren Zentralpart noch spielen sollte. Besonders im Verlauf der europäischen Integration waren immer mehr und immer wichtigere Kompetenzen, die man früher als eindeutig und geradezu identitätsprägend national eingestuft hätte, an die beständig enger zusammenrückende Union der Staaten der Europäischen Gemeinschaft abgegeben worden. Doch nicht allein das: Neben der Europäischen Union als der supranationalen ist immer stärker und intensiver die internationale Ebene mit ihren mannigfaltigen Akteuren und Entscheidungsträgern in Rechnung zu stellen - und sind auch hier entsprechende Regelungs- und Kompetenzverluste des Staates zu registrieren. Im Fokus der Debatte stand dabei von jeher die WTO, deren Entscheidungen in der Tat und ganz unbestritten tief in die nationalen Rechtsordnungen einschneiden können. Am empfindlichsten aber empfand man zumindest in Deutschland den Verlust einer der vermeintlich härtesten und essentiellsten staatlichen Hoheiten, nämlich der Währungshoheit. Das lag womöglich nicht nur an der besonderen Symbolträchtigkeit und war von daher auch nicht nur als Phantomschmerz zu verbuchen. Die Befürchtung, dass es sich bei dieser Aufgabe um einen geradezu existentiellen Vorgang mit unabsehbaren Folgen gehandelt haben könnte, ist bei weitem nicht endgültig zerstreut. Jede Nachricht aus Griechenland verschafft ihr in diesen Tagen neue Nahrung. 
Mit supra- und internationaler Expropriation des alten Nationalstaates hatte und hat es aber noch nicht sein Bewenden. Denn als zweites Merkzeichen, wenn nicht gleich Menetekel, trat spätestens seit den 1980er Jahren die nicht selten europarechtlich induzierte Privatisierung auf den Plan. Dieser umfassende, in sich vielfach zu differenzierende Prozess bildet sozusagen den zweiten Dezimierungsfaktor vermeintlicher staatlicher Omnipotenz. Aufgabenprivatisierung bedingt Staatlichkeitsverlust nicht durch Abgabe der hoheitlichen Aufgabe an andere Hoheitsträger, sondern durch ihre Transformation in eine von Privaten erbrachte Marktleistung. Im Telekommunikationsbereich war das mit Händen zu greifen und bis in die Grundrechtsdetails zu verfolgen. Je mehr private und marktrechtsförmige Erledigung, desto weniger Staat - auf diese (freilich viel zu einfache) Formel wurde es gerne gebracht. Und selbst dort, wo jedenfalls formell die nationalstaatliche Gesetzgebungs- oder Verwaltungshoheit ungebrochen fortbestand, ließen sich zum Teil besorgniserregende Vorgänge einer zunehmenden Entformalisierung, sei es des Legislativverfahrens (Stichwort: Atomkonsens), sei es der Verwaltungspraxis (Stichwort: informales oder kooperatives Verwaltungshandeln), ausmachen. Weicher Konsens statt harten Rechtsbefehls, der Staat nicht hoheitliche Anstalt, sondern Partner, der Bürger nicht gewaltunterworfenes Subjekt, sondern (möglichst Englisch sprechender) Kunde, die Gesellschaft eher ein erweiterter Freizeitpark als eine politische Schicksalsgemeinschaft - so ungefähr bot sich das neue Gesamtbild dar.

In der Summe ergab sich in den Augen vieler der nur schwer widerlegbare Eindruck wenn schon nicht einer vollkommenen Auflösung, so doch einer unaufhaltsamen Auszehrung des Staates durch sukzessiven Aufgabenverlust mit entsprechend sinkender Gesamtrelevanz für die politisch-gesellschaftliche Ordnung: einerseits durch Etablierung anderer Hoheitsträger auf supranationaler Ebene, andererseits durch gezielt herbeigeführte oder ungezielt eingetretene Abwanderung vormals staatlicher Agenden auf den Markt oder sonstige Selbstregulationsmechanismen nationaler wie transnationaler Art.

\section{Wiederaufnahme der Debatte}

Zwei Ereignisse, die miteinander weder in einem kausalen noch systematischen Zusammenhang stehen, haben den Staat jedenfalls dem ersten Anschein nach zurückgebracht, um auf eine vielzitierte englische Publikation (,,Bringing the State Back In") anzuspielen. Durch die anhaltende Wirtschafts- und Finanzkrise der letzten Jahre sowie den Paukenschlag des Lissabon-Urteils des Bundesverfassungsgerichts wurde der Staat auf jeweils ganz eigene, aber zunächst einmal 
sehr eindrucksvolle Weise auf die Hauptbühne des Geschehens zurückgeholt, sozusagen erneut ins Rampenlicht gestellt. Seit 2007 hat die Banken-, Finanzund Wirtschaftskrise den „Ruf nach dem Staat in lange nicht gehörter Intensität erschallen lassen“ (Werner Heun). Und er wurde erhört. Es war die Bundesrepublik Deutschland (und nicht transnationale zivilgesellschaftliche Netzwerke oder globale Wirtschaftskonsortien), die der deutschen Unternehmenstochter eines amerikanischen Automobilkonzerns mit Milliardenbeiträgen in Gestalt von Überbrückungskrediten und Kreditbürgschaften unter die Arme griff und eine mittlerweile wieder aufgelöste Opel-Treuhandgesellschaft gründete. Es war vor allem die Bundesrepublik Deutschland (und nicht die Europäische Union, auch nicht der Internationale Währungsfonds (IWF) oder die WTO), die innerhalb dieses ohne Zweifel größten und gefährlichsten Krisensektors gleich mehrere Banken mit kurzfristigen staatlichen Garantien stützte, ihnen Liquiditätserleichterungen verschaffte und in Gestalt einer per Eilgesetz etablierten Anstalt zu Finanzmarktstabilisierung staatliches Kapital im Wert von über $€ 100$ Mrd. bereitstellte. Eine der Banken, die Hypo Real Estate, wurde inzwischen vollständig verstaatlicht, bei der Commerzbank beläuft sich der Staatsanteil auf 25 Prozent plus eine Aktie. Auch an der Westdeutschen Landesbank ist die Bundesrepublik Deutschland nun in erheblichem Umfang beteiligt. Wer solche Vorgänge vor zehn Jahren zu prognostizieren gewagt hätte: Bankenverstaatlichung in Deutschland unter einer CDU-geführten Bundesregierung, wäre schlicht nicht ernstgenommen worden. Heute aber ist der Staat Bankier, wenn auch nur „wider Willen" (Joachim Wieland) - aber bis auf weiteres bleibt er es und steht vor der Frage, wie er mit dieser ungewohnten Rolle umgehen soll.

Den zweiten Hauptschauplatz für ein re-entry des Staates bildet die Beschränkung der Fortentwicklungsmöglichkeiten der Europäischen Union, wie sie aus Sicht des deutschen Grundgesetzes in der Interpretation des Bundesverfassungsgerichts erfolgt ist. Denn mit einem von manchen nicht für möglich gehaltenen Nachdruck fixierte das Gericht in seinem Lissabon-Urteil vom Juni 2009 Grenzen weiterer Integrationsschritte. Dem Urteil zufolge ermächtigt Art. 23 GG (nur) zur Beteiligung und Entwicklung einer als Staatenverbund ausgestalteten Europäischen Union, deren Grundordnung aber allein der Verfügung der Mitgliedstaaten unterliege. Ausgeschlossen ist demzufolge ein Standard der europäischen Integration, der den Mitgliedstaaten keinen ausreichenden Raum zur politischen Gestaltung ihrer wirtschaftlichen, kulturellen und sozialen Lebensverhältnisse mehr belässt. Ein klarer Riegel wird zudem dem Übergang des Staatenverbundes hin zu einem europäischen Bundesstaat vorgeschoben, den sich bis- 
lang viele in Gestalt eines inkrementalen oder iterativen Prozesses vorstellen konnten. Ein solcher Identitätswechsel der Bundesrepublik Deutschland kann dem Bundesverfassungsgericht zufolge nicht auf Grundlage und im Rahmen des Grundgesetzes stattfinden. Diese setze vielmehr einen Akt der Verfassunggebung voraus, für dessen Genese Art. $146 \mathrm{GG}$ den Weg weise. Eine ungewöhnlich ausführlich ausfallende, grundgesetzlich nicht verankerte und geradezu schematisch-katalogartig ausfallende Liste angeblich unaufgebbarer zentraler Staatsaufgaben rundet das Bild eines Urteils ab, dem in kritischer Intention vorgehalten wurde, so etwas wie ein „Recht auf Staatlichkeit“ generiert zu haben - von sonstiger, oft ungewöhnlich scharfer und beileibe nicht immer unberechtigter Kritik an Duktus, Fundierung, Programmatik und Stringenz der Entscheidung einmal ganz abgesehen.

\section{Unverzichtbare Ordnungs- und Stabiliserungsleistungen}

Der Staat, so hat es den Anschein, ist also zurück auf der Agenda - aber war er eigentlich jemals fort? Weitgehend entschwunden oder schlicht irrelevant geworden war er womöglich für jene, deren Aufmerksamkeitsspektrum ganz auf Gebiete wie das internationale Wirtschaftsrecht, das Internet, das transnationale Copyright oder ähnliche Felder gerichtet war oder die sich ohnehin vornehmlich in den luftigen Höhen hochspekulativer Theorien über die Autopoiesis des Rechts mit seinen Hyperzyklen, rekursiven Schleifen und Netzwerkstrukturen bewegt hatten. Von dieser vielleicht nicht allzu repräsentativen Warte aus sah man in der Tat keine Hierarchien mehr, sondern nur noch Heterarchien, kannte statt (durchaus dezisionistischer) heteronomer Rechtssetzung nur noch die Eigenrationalitäten merkwürdig subjektloser autonomer Rechtsregimes; von hier aus erblickte man im globalen Recht einschließlich internationaler gerichtsförmiger Spruchkörper die Zukunft und erspürte in global governance den Zug der Zeit. Nationale Gesetzgebung, Verwaltung oder Judikatur erschienen da als eher oldfashioned, der Nationalstaat insgesamt als bedauernswertes Relikt einer im Grunde zum Untergang verurteilten Epoche.

Im Gegensatz hierzu hatten allerdings nüchterne Naturen mit etwas stärkerer Bodenhaftung beharrlich festgehalten, dass die Gesamtheit hoheitlicher Ordnungs- und Leistungsaufgaben nach wie vor ganz überwiegend nationalstaatlich geprägt ist. Das gilt, bei allen grenzüberschreitenden Aspekten und supranationalen Einwirkungen, für die Gefahrenabwehr ebenso wie für die Strafverfolgung, für die Erbringung von Sozialleistungen wie für die Versorgung mit Kindergärten, es gilt für die staatlichen Schulen wie für die Finanzämter, es gilt für die 
Durchsetzung der Wehr- und Ersatzdienstpflicht nicht anders als für den nach wie vor ganz überwiegend von deutschen Gerichten gewährten Rechtsschutz. Bei der Sicherung des inneren Friedens, der Realisierung staatlicher Schutzpflichten, der Justizgewährleistung oder der Garantie eines materiellen Existenzminimums für alle in Deutschland lebenden Menschen - ganz unabhängig von ihrer Nationalität - ist von den in akademischen Oberseminaren und ihnen vergleichbaren Literaturgattungen gern zitierten Prozessen der Transnationalisierung, Globalisierung und Selbstregulierung relativ wenig zu spüren. Zugegebenermaßen fehlt hier, gerade bei präziser politikwissenschaftlicher und juristischer Betrachtung, durchweg der Charme des visionär Ausgreifenden, der juristisch-politischen Weltformel, des ganz neue Perspektiven eröffnenden Wurfes, es mangelt am kühnen Ausgriff ins Große und Ganzheitliche. Es sind eher die Mühen der Ebene zu bewältigen, nicht die Ausblicke vom Berggipfel zu genießen. Doch nur so schreitet man voran. Und nur so kann man auch die nationalen, supranationalen und internationalen, die wirtschaftlichen, politischen und ökonomischen Rahmenbedingungen adäquat erfassen, die die Voraussetzungen für staatliches Handeln bilden und es in vielfältiger Weise beeinflussen.

\section{Lissabon und Wirtschaftskrise: Staatstheorie vor neuen Herausforderungen}

Wenn der Staat also möglicherweise wieder etwas stärker in den Blickpunkt gerückt ist, so ließe sich das vielleicht für eine etwas differenziertere Rückbesinnung auf Leistungsfähigkeit und Leistungsgrenzen, auf Macht und Ohnmacht staatlicher Handlungsmöglichkeiten nutzen. Man erläge ja einem schweren Irrtum, wenn man die hier plakativ konstatierte „Rückkehr“ umstandslos interpretieren würde als Re-Inthronisation eines Staates im Sinne einer omnipotenten und autonomen Größe mit praktisch unbegrenzter Regelungskompetenz und Steuerungsfähigkeit. Über eine solche Allmacht verfügte der Staat in seiner mehrhundertjährigen Geschichte ohnehin noch nie. Souveränität hat niemals Omnipotenz, Omnipräsenz und Omnikompetenz bedeutet. Selbst auf dem Höhepunkt des absolutistischen Fürstenstaates waren doch immer Faktoren des „Nichtabsolutistischen im Absolutismus“ (Gerhard Oestreich) wirksam. Stets ging es bei der Entfaltung staatlicher Souveränität um die relative, im Laufe der Geschichte mehr oder minder große Unabhängigkeit von gewichtigen sozialen Kräften und konkurrierenden politischen Mächten, unter demokratischen Vorzeichen dann vor allem auch um die Koppelung von Volkssouveränität und Rechtsstaatlichkeit unter dem Dach einer Verfassung, die auf der „verfassunggebenden 
Gewalt des Volkes“ beruht, von der die Präambel des Grundgesetzes spricht. Souveränität bedeutet begrifflich Verdichtung und Monopolisierung von Herrschaftsbefugnissen in einem Punkt, rechtlich mehr oder minder virtuelle Letztentscheidungsgewalt des Staates und seine Kompetenz-Kompetenz bei der Aufgabenerfüllung. In Bezug auf die europäische Integration hat das Bundesverfassungsgericht dem im Lissabon-Urteil eine klare, wenn auch in der Wissenschaft stark umstrittene Konkretion verliehen: Unzulässigkeit eines europäischen Bundesstaates, Mitgliedstaaten als Herren über die Verträge, Austrittsrecht, ein Kern der Staatsverfassung als unantastbares Reservat.

Das klingt allerdings durchweg schneidiger als es ist. Denn natürlich lassen sich Supranationalisierung (wie auch Internationalisierung) nicht bannen. Dafür sind diese Prozesse schon viel zu weit und durchweg mit dem Segen des Gerichts fortgeschritten. Andererseits werden wir schon aus rein politischen und ökonomischen Gründen auf absehbare Zeit von einem europäischen Bundesstaat entfernt bleiben. Doch ungeachtet dessen bestimmt sich schon heute in vielen Aspekten die konkrete Gestalt der Rechtsordnung in einem Staat nicht mehr allein nach der Rechtsordnung dieses Staates. Nur sollte man eben nicht so tun, als gelte das mittlerweile flächendeckend und überall in gleichem Intensitätsgrad. Zudem wird der Staat als zentraler Akteur durch diese Prozesse ja keineswegs überflüssig oder praktisch inexistent - schließlich ist er es ja in Ermangelung entsprechender internationaler oder supranationaler Behörden und Institutionen, der diese Einwirkungen um- und durchsetzen muss. Wenn transnationale Hoheitsgewalten neben den Staat treten, erhöht sich die Komplexität des Geschehens und der Gesamtarchitektur, die in der Bundesrepublik Deutschland wegen der föderalen Struktur ohnehin schon kompliziert genug ist. Die Schulung in Bundesstaatlichkeit erleichtert zugleich das Verständnis für die komplexe Mehrebenenstruktur der EU und ihrer Mitgliedstaaten. Hinzutritt, dass die Staats- und vor allem die Verwaltungsorganisation der Bundesrepublik Deutschland (und nicht erst sie) seit jeher durch ein besonders hohes Maß an verselbständigten Verwaltungseinheiten gekennzeichnet ist. Was in aktuellen, eher politikwissenschaftlich geprägten Kontexten kritisch als vermeintlich junges Phänomen der "Zerfaserung" des Staates angesprochen wird, stellt insofern seit jeher gleichsam die Normallage dar - auch wenn diese gravierende verfassungs- wie verwaltungsrechtliche Fragen aufwirft.

Und wie steht es bei der Privatisierung? Hier gewinnt man seit geraumer Zeit den Eindruck, dass die Markteuphorie mittlerweile deutlich nachgelassen hat ohne dass die damit verbundenen Flexibilisierungsleistungen und die Effizienz- 
und Freiheitsgewinne, die mit den einschlägigen Maßnahmen erzielt worden sind, unterschätzt oder kleingeredet werden sollten. Auch ändert sich dadurch, dass der Staat in der Finanzkrise einige Banken übernommen und gewaltige finanzielle Stützungsanstrengungen übernommen hat, nicht gleich das Gesamtgefüge der Wirtschaftsordnung - einmal ganz abgesehen davon, ob und inwieweit das Handeln aus einer Position neuer Stärke oder nicht eher aus nackter Not erfolgte, aber mit einem kräftigen Schuss an Symbolik versehen wurde. Wichtiger ist da schon die ganz grundsätzliche Einsicht, dass ein Markt überhaupt erst einmal konstituiert werden muss und auf einen entsprechenden Rechtsrahmen angewiesen ist. Und für die speziellere Frage nach der Rolle des Staates angesichts vergangener und vielleicht zukünftiger Privatisierungsvorgänge ist der gern übersehene Umstand von weit reichender Bedeutung, dass Privatisierung keineswegs stets vollumfänglich Entstaatlichung bedeutet. Man muss sich von der Vorstellung lösen, Privatisierung führe zum ersatzlosen Fortfall von Verwaltungstätigkeit oder staatlicher Ingerenz. Was eintritt, ist ein Wandel der Aufgabenstrukturen: eher ein Umbau als ein Abbau staatlicher Verwaltung, wie er beispielsweise im Bereich von Post und Telekommunikation zu registrieren war. Gewährleistungsstaat im Allgemeinen und Regulierungsverwaltung im Besonderen wurden so zu vergleichsweise geläufigen (wenn auch nicht immer definierten) Bezeichnungen, unter denen die vielfältigen Prozesse in der Rechtswissenschaft diskutiert und dogmatisch eingefangen werden (sollten). Die Staatswissenschaften täten gut daran, auch und gerade solche Phänomene nicht zu übersehen. Manch steile These könnte so vielleicht durch materialgesättigte Erklärungskonzepte ersetzt werden.

Man sollte das durch Wirtschaftskrise und Lissabon-Judikatur möglicherweise neu erwachte Interesse an Staat und Staatlichkeit als Chance für eine verstärkte Konzentration auf eine Staatstheorie begreifen, die diese Konstellation unter den Bedingungen des 21. Jahrhunderts mit Realismus und analytischer Kraft untersucht und so die Lage des Staates jenseits nebelhafter Visionen oder verfrühter Nachrufe etwas genauer in den Blick nimmt. Wir müssen uns, so ließe sich vielleicht resümieren, nicht vom Staat verabschieden, sondern nur von einem zu einfachen Bild, das manche sich von ihm gemacht haben. Darin liegt die eigentliche Herausforderung.

\section{Staatswissenschaften und Staatspraxis}

Die Autoren des von uns herausgegebenen ZSE-Themenheftes haben sich dieser Herausforderung gestellt, wobei der unterschiedliche disziplinäre Zugriff und das 
heterogene Verhältnis zur Staatspraxis sich als produktive Anregungen erwiesen. Während die jeweilige disziplinäre Herkunft die theoretischen, empirischanalytischen und methodischen Vorgehensweisen dokumentierte, mithin die Reichweite und den Ertrag der die Staatswissenschaften konstituierenden Disziplinen widerspiegelte, war der Zugang zur Staatspraxis durch eine interessante Mischung aus Nähe und Distanz gekennzeichnet: Einige Autoren konnten Erfahrungen als „handelnder Akteur“ einbringen, andere führte die (in Teilen bewusste) Distanz zu Erkenntnissen, die nicht selten in anregende „Grenzüberschreitungen“ mündeten. Dies gilt nicht nur für jene Beiträge, die die „Ehrbarkeit des Staates“ oder die „Herausforderungen des Naturrechts“ in das Zentrum Ihrer Ausführungen stellten, sondern auch für (nur vermeintlich periphere) Ansätze wie den, der den „Staat als Glücksmaximierer“ diskutierte.

\section{Nationalstaat und Europäische Union}

Folgt man der von den Herausgebern angebotenen deduktiven Logik, so wird man nach Durchsicht der Beiträge zum Themenbereich „Nationalstaat und Europäische Union - Lissabon und die Folgen" eine staats- wie europawissenschaftlich interessante Auseinandersetzung wahrnehmen. Sie richtet sich in Ergänzung der in der Einführung angebotenen Orientierungen zum einen auf die alles andere als gradlinige Entwicklung des Verhältnisses der Europäischen Union zu ihren Mitgliedstaaten, sucht zum zweiten die Konsequenzen des „Lissabon-Urteils“ des Bundesverfassungsgerichts zu umreißen und wird aufgrund neuerer Entwicklungsprozesse schließlich zu einem Testfall dessen, was von den handelnden Akteuren heute als sinnvoll und ,machbar“ einschätzt wird. Letzteres richtet sich vor allem auf die im Zuge der streitigen Finanzhilfen für Griechenland erwartbaren erneuten Vertragsänderungen, die insbesondere darauf zielen, ein ,zweites Athen" zu vermeiden, zumal auch die Haushalte Portugals, Spaniens und Italiens vergleichbare Verwerfungen beinhalten. Dies mag erklären, weshalb sich die deutsche Bundeskanzlerin damit durchzusetzen suchte, bilaterale Hilfen von Euro-Staaten nur für den Fall vorzusehen, dass alle anderen Optionen ausgeschöpft wurden und zudem eine substantielle Beteiligung des IWF gewährleistet ist. Mit der jetzt unter Vorsitz des Ratspräsidenten Van Rompuy eingesetzten Arbeitsgruppe geht es vor allem um eine Verschärfung des EU-Stabilitätspakts bis hin zu der von der Kanzlerin eingebrachten, allerdings strittigen Konsequenz eines etwaigen Ausschlusses stabilitätsgefährdender Länder aus dem EuroRaum. Zudem wird angestrebt, dass die Arbeitsgruppe sich nicht nur aus Repräsentanten der die Euro-Gruppe bildenden Staaten zusammensetzt, sondern auch 
weitere Mitgliedstaaten, die EZB und die Kommission einbezieht. Schließlich strebt man an, die auch vom Präsidenten der EZB, Trichet, eingeforderte Verantwortung zur gegenseitigen Überwachung der Wirtschaftspolitiken innerhalb der Euro-Gruppe und des Europäischen Rats umzusetzen.

Im Übrigen machen die Bedingungen für etwaige Finanzhilfen deutlich, dass die Staats- und Regierungschefs der Euro-Gruppe bilaterale Kredite in der Tat nur als ultima ratio ansehen. Die Mittel fließen nur dann, wenn Griechenland am Markt als nicht mehr kreditwürdig eingeschätzt wird; zudem dürfen die in diesem Fall gewährten Kredite kein Subventionselement enthalten, müssen mithin zu hinreichend hohen Zinsen gewährt werden. Auch gilt, dass jeder Beschluss zur Hilfestellung in der Euro-Gruppe einstimmig erfolgen und eine Beurteilung der EZB einbeziehen muss, die Beteiligten sich also eine Veto-Position vorbehalten. Im Ergebnis steht ein Kompromiss, der die hochspekulativen Märkte beruhigen, aber auch einer Denk- und Interpretationsrichtung entgegentreten sollte, die von einer allmählichen Zweiteilung der Europäischen Union in North and South of Paris spricht. Dahinter verbirgt sich ein zunehmendes Bewusstsein, dass die nord- und mitteleuropäischen Mitgliedstaaten der Europäischen Union anderen Rationalitätsvorstellungen, Organisationsprinzipien und Verhaltensmustern folgen als dies für einige der südeuropäischen Mitgliedstaaten erkennbar ist. Verstärkt durch Diskussionen, die den EU-Beitritt Rumäniens und Bulgariens inzwischen als ,erkennbar zu früh“ einschätzen, verdichten sich hier Wahrnehmungen, die eine europapolitische Solidarfunktion der Beteiligten zwar nicht in Abrede stellen, sie allerdings für zunehmend voraussetzungsvoll halten. Im Übrigen verweist die laufende Diskussion um Griechenland-Hilfen auf die die Akteure seit bald 40 Jahren beschäftigende Frage nach der Bildung einer europäischen Wirtschaftsregierung. So fanden sich bereits im Abschlussbericht der vom damaligen luxemburgischen Premier- und Finanzminister Pierre Werner geleiteten Sachverständigengruppe zur Verwirklichung der Wirtschafts- und Währungsunion bis 1980 Vorstellungen, die den heutigen Diskussionen ähneln: „Da hinsichtlich der Verwirklichung des Wachstums- und Stabilitätsziels zwischen den Mitgliedstaaten auch deutliche Unterschiede bestehen, ist ohne eine effektive Harmonisierung der Wirtschaftspolitik die Gefahr der Entstehung von Ungleichgewichten weiterhin gegeben." ${ }^{2}$ Zwar wurde diesen Vorstellungen in Ihrer Gesamtheit - und vor allem operativ - nicht gefolgt, doch kam es im Verlauf des

2 Pierre Werner et al.: Report on the Realisation by Stages of Economic and Monetary Union, in: Bulletin of the European Communities, 11/70 (1970), 5-29. 
weiteren Integrationsprozesses zu wichtigen Einzelschritten, von der Bildung der sog. „Währungsschlange“, also des Europäischen Wechselkursverbunds von 1972, über die Schaffung des Europäischen Währungssystems (EWS) 1979 und den ab 1995 in Angriff genommene Binnenmarkt bis hin zu der 1999 vereinbarten Obergrenze für die öffentliche Verschuldung.

Eine Angleichung oder auch nur Abstimmung von Wirtschafts-, Haushalts- und Beschäftigungspolitiken verband sich damit freilich nicht, da die in Aussicht genommene einheitliche Währung als ausreichender Katalysator für die wirtschaftliche und politische Union eingeschätzt wurde. Der Vertrag von Maastricht folgte dem dann aufgrund der vereinbarten Architektur: hier ein gemeinschaftliches Standbein mit einer unabhängigen, der Preisstabilität verpflichteten EZB, dort eine stärkere Abstimmung in der Wirtschaftspolitik unter Achtung der alleinigen Befugnisse der Mitgliedstaaten. 1997 schließlich wurde der Stabilitäts- und Wachstumspakt verabschiedet, der ein gedeihliches Nebeneinander von Geld-, Finanz- und Wirtschaftspolitiken ermöglichen sollte. Seitdem bemühen sich die Finanzminister im Rahmen der Euro-Gruppe um eine verbesserte wirtschaftpolitische Abstimmung, deren Wirkungsweise außerhalb von Krisen als durchaus ausreichend eingeschätzt wurde. Erst seit dem Jahr 2008, also mit Einsetzen der gegenwärtigen Finanz- und Wirtschaftskrise, erfuhr die Diskussion eine neue Qualität, die sich jetzt mit erweiterten Strategien zur Förderung der europäischen Wachstums- und Wettbewerbsfähigkeit verbindet. Bislang scheint sich dabei die deutsche Seite insofern durchzusetzen, als man hier die Staats- und Regierungschefs der 27 EU-Staaten als „Wirtschaftsregierung“ begreift, die nach dem Lissabonner Vertrag die für die Entwicklung der Union „erforderlichen Impulse“ gewährleisten sollte. Der Vertrag selbst beschränkt sich in Art. 121 AEUV auf die Möglichkeit einer „Verwarnung“ wirtschaftspolitisch säumiger Regierungen. Allerdings ist die Diskussion damit erkennbar nicht beendet: Während die Bundesregierung eine lediglich verbesserte wirtschaftspolitische Koordination anstrebt, zielt Frankreich weiter auf eine enge materielle und wie zeitliche Verzahnung von Stabilitätspakt wie den im Zuge des Projektes „Europa 2020“ geplanten wirtschafts- und strukturpolitischen Empfehlungen. ${ }^{3}$

Im Übrigen ist nicht wirklich absehbar, dass die schwierige Ratifizierung des Lissabon-Vertrages ein nachhaltiges Umdenken auf Seiten der europäisch wie

3 Europäische Kommission: Europa 2020. Eine Strategie für intelligentes, nachhaltiges und integratives Wachstum, Mitteilung der Kommission, KOM (2010) 2020, Brüssel, 2010, http://ec.europa.eu/growth andjobs/pdf/complet_de.pdf. 
nationalstaatlich Handelnden bewirkt hätte. Zwar bestätigt man allenthalben eine gewisse Entfremdung zwischen den europäischen Einrichtungen und dem Souverän, doch werden daraus kaum auch operative Konsequenzen gezogen. Während die Besetzung der im Vertrag vorgesehenen europäischen Spitzenämter mit Herman Van Rompuy und Baroness Ashton darauf hindeutet, dass man die angestrebte „Sichtbarkeit“ nun denn doch nicht gegen die (größeren) Nationalstaaten ausfallen lassen wollte, wird auch in der Weiterentwicklung wichtiger Aufgabenund Politikfelder sowie bei der Schaffung neuer Einrichtungen ein seit langem erkennbares Grundmuster deutlich.

So finden sich mit Blick auf die bereits angesprochene Wachstumsstrategie „Europa 2020“ nicht nur ein déjà-vu-Effekt hinsichtlich der reputationsschädigend gescheiterten sog. „Lissabon-Agenda“, in deren Verfolgung die EU sich bekanntlich bis zum Jahr 2010 zur ,wettbewerbsfähigsten Region der Welt“ machen wollte, sondern auch Zielvorstellungen, die auf beträchtlichen Widerstand unter den EU-Mitgliedstaaten stoßen. Hier sind es erneut die Nord- und Mitteleuropäer, die die von der Kommission beabsichtigte Festlegung von Bildungszielen und Vorgaben für die Armutsbekämpfung materiell wie im Verfahren kritisieren. Formal wird darauf verwiesen, dass die Kommission damit die im Vertrag von Lissabon aufgeführten Kompetenzen überschreitet, materiell gelten die Bedenken den vorgestellten „fünf Kernzielen“ der „2020-Strategie“: Verringerung der Zahl der von Armut betroffenen Menschen in der EU um 20 Mio., Erhöhung des Anteils von Hochschulabsolventen in der Gruppe der Dreißig- bis VierunddreiBigjährigen von heute durchschnittliche $31 \%$ auf künftig $40 \%$, Anhebung der Beschäftigungsquote der Zwanzig- bis Vierundsechzigjährigen von $68 \%$ auf $75 \%$ und schließlich Anstieg der Forschungsausgaben auf $3 \%$ des BIP. Während die letztbenannten Ziele wohl insofern konsensfähig sind, als sie sich in fast allen politischen Absichtserklärungen der Mitgliedstaaten finden, stehen die „Vorgaben“ für das Bildungsniveau und den Abbau der Armut nach Angaben der Beteiligten erheblicher Kritik. Die hier von der Kommission verfolgte Strategie, konkrete Empfehlungen für die einzelnen Mitgliedstaaten zu formulieren, einen Zeitrahmen für ihre Umsetzung vorzugeben und bei Verfehlen der Vorgaben Verwarnungen auszusprechen sucht, muss in der Tat erstaunen. Zudem erinnert das vom Kommissionspräsidenten Barroso vorgeschlagene Verfahren, sich zunächst auf grundsätzliche Ziele einer solchen Wachstumsstrategie zu einigen, um dann über Detailziele in den darauf folgenden Monaten zu verhandeln, an den schon mehrfach eingeschlagenen Weg, über breit formulierte Absichtserklä- 
rungen eine self-fulfilling prophecy in Gang zu setzen, um so die eigene Bedeutung und das Steuerungspotential gegenüber den Mitgliedstaaten zu erhöhen.

Auch der Aufbau des künftigen Auswärtigen Dienstes der EU dokumentiert neben mangelnder Lernfähigkeit - jene sattsam bekannten Auseinandersetzungen zwischen den Beteiligten, die die „Völker Europas“ dem Europäisierungsprozess durchaus entfremden. So ist weiterhin strittig, wie sich der Auswärtige Dienst zusammensetzen wird und wer ihn letztlich führt, ob und wie man die absehbaren Doppelstrukturen der Außenvertretung abbauen könnte und wer schließlich - mit Blick auf materielle Politiken - die Federführung übernehmen soll: Nationalregierungen, die Kommission oder die „Hohe Vertreterin“. Zudem ist umstritten, wer das Weisungsrecht gegenüber den künftigen „EUBotschaftern" ausübt und selbst das Verfahren zur Ernennung dieser Botschafter bleibt offen. Immerhin geht es nach Vorstellungen der Kommission mit dem Auswärtigen Dienst um bis zu 1.200 Diplomaten im höheren Dienst, unter Einschluss von Ortskräften wird (auf der Basis von heute 137 Delegationen in Drittländern) von insgesamt etwa 8.000 Mitarbeitern ausgegangen. Selbst die formale Beschlussfassung zur Gründung des Dienstes unterliegt geradezu EU-typischen tagespolitischen Aktualitäten und „Anpassungsprozessen“: So war ursprünglich geplant, die Begründung des Dienstes im April 2010 vorzunehmen, also noch vor der Wahl in Großbritannien, da man im Fall eines Regierungswechsels eine Blockade befürchtet. Auch bleibt mit Blick auf das legitimatorische Potential solch strukturell bedeutsamer „Innovationen“ darauf hinzuweisen, dass der Souverän, vertreten durch das Europäische Parlament, bislang nicht am Gründungsverfahren beteiligt ist; das EP erwägt deshalb, seine Vorstellungen über die Haushaltsberatungen und das Personalstatut einzubringen.

Im Ergebnis fällt es schwer, in der derzeitigen Entwicklungsphase der Europäischen Union jene Stabilisierung und Konsolidierung auszumachen, auf die man sich während und nach der langwierigen Ratifikation des Vertrages von Lissabon zu einigen schien. Statt das noch immer problematische Verhältnis zum Souverän in das Zentrum der Bemühungen zu stellen, werden neue Auseinandersetzungen erkennbar, die das europäische Projekt sicher nicht gefährden, aber seiner erweiterten Legitimation und unabwendbaren Professionalisierung entgegenzustehen drohen. Ob Krisenbewältigung, Verfahrensvereinfachung oder institutionelle Reform, es finden sich unverändert Verhaltensmuster, die eher von einem Gegeneinander denn einem Miteinander der europäischen und der nationalstaatlichen Ebenen geprägt scheinen. Hier sollten sich EU-Einrichtungen inzwischen doch in einer deutlichen „Bringschuld“ sehen, die vor allem darin bestehen könn- 
te, sich eher in einer den „Völkern Europas“ dienenden Rolle denn als „Innovationsmotor" zu verstehen, dessen legitimatorische Basis und operative Kompetenz fragwürdig bleiben. Hinzu treten die restringierenden Wirkungen der LissabonEntscheidung des Bundesverfassungsgerichts.

\section{Der Staat in der Krise}

Ließen die Beiträge zum Verhältnis von Nationalstaat und Europäischer Union bereits erkennen, dass sich - bei durchgehender positiver europäischer Grundhaltung - eine Reihe von Vorbehalten stellen, die sich entweder mit dem Selbstverständnis oder dem faktischen Wirken der europäischen Einrichtungen verbinden, erbrachten auch die Beiträge zum Themenbereich „Der Staat in der Krise“ eine Reihe von Monita: Während Wirtschaftshistoriker den wirtschaftswissenschaftlichen Erkenntnisprozess wie die dabei meist eingesetzte Methodik kritisieren und aufgrund der Marginalisierung von Faktoren wie menschliches Verhalten, Moral und Naturrecht den Wirtschaftswissenschaften nicht weniger als empirisches Versagen und eine Beschädigung ideeller Werte vorwerfen, wird dies bei Ergänzung um Kategorien der politischen Ökonomie noch durch die Aussage zugespitzt, dass das Vertrauen auf die Zwangsläufigkeit wirtschaftlich-technokratischer Integrationsprozesse zur politische Lebenslüge der Gemeinschaft geworden sei und dies in hohem Maße auch für die heutige Europäische Union gelte; die systematische Vernachlässigung des Verhältnisses zum europäischen Souverän könnte danach zu einer Schlüsselfrage der weiteren Entwicklung werden.

Darüber hinaus stehen die erkennbaren Muster der Krisenbewältigung im Vordergrund, wobei die künftige Rolle und Funktion des Staates im Rahmen von Wirtschafts- und Finanzkrisen sowie vor allem das eingesetzte Instrumentarium einer kritischen Würdigung unterzogen werden. Sehen die einen den Staat als gleichsam „Aktionär der letzten Instanz“, der im Gefolge der Finanzkrise eben zum Bankier wider Willen geworden sei, fragen andere nach der „Ehrbarkeit“ eben dieses Staates angesichts des größten Konjunkturprogramms der deutschen Geschichte und der sich damit verbindenden Folgewirkungen für die öffentlichen Haushalte. Selbst das eingesetzte Instrumentarium wird in weiten Teilen kritisch beurteilt; dies gilt für den Ruf nach verstärkter Regulierung ebenso wie für jene Steuerung der Staatsverschuldung, auf die man sich seitens der Politik als Ergebnis der Empfehlungen der Föderalismuskommission II einigte. Schließlich kommt es zu einer kompakten Evaluation der eingesetzten Konsolidierungsstrategien samt sich damit verbindender Empfehlungen, die auch ausgabenseitige Überprüfungen, eine in diesem Kontext zu verfolgende politische road map, ein 
erweitertes politisches commitment und schließlich nur vermeintlich technische Hinweise, etwa die Einführung von Doppelhaushalten oder die Vornahme von Sicherheitsabschlägen, einbeziehen.

Im Ergebnis erweist sich, dass die ablaufende Krise nicht mehr nur als gleichsam situatives Problem, sondern als grundlegende Herausforderung begriffen werden sollte, die eine Überprüfung rechtlicher Regelungen, erweiterte Steuerungsmodi, einen veränderten institutionellen Rahmen sowie prozessuale und materielle Verbesserungen nahelegt. Dabei wird man zwischen dem, was ,nach außen“ und dem, was „nach innen“ vorgeschlagen wird, unterscheiden können.

Nach außen geht es vor allem um eine als dringend erforderlich bezeichnete Abstimmung nicht nur nationalstaatlicher Geld- und Fiskalpolitiken, sondern auch und gerade jener internationalen Steuerungs- und Stabilisierungsleistungen, wie sie im Rahmen der seit Ende des Zweiten Weltkrieges aufgebauten Architektur der Internationalen Organisationen erbracht werden. Die Erkenntnis, nach der die Entwicklung der Finanzmärkte ohne grenzüberschreitende Regulierung eine inzwischen „systemische Bedrohung“ darstellt, findet in diesem Kontext breiten Konsens. So geht es mit Blick auf den Bankensektor nicht mehr nur um eine wirksame Aufsicht und Steuerung, sondern auch um die finanzielle Mitverantwortung für die von diesem Wirtschaftsbereich ausgehenden Risiken. Vor diesem Hintergrund erstaunt, dass das seit langem geplante Gesetz zur Finanzmarktregulierung noch immer nur in Umrissen erkennbar ist. Zwar besteht weitgehender Konsens darin, über eine Bankenabgabe Lehren aus der Finanzkrise zu ziehen, um zu verhindern, dass der Staat auch künftig im Krisenfall Banken mit Steuergeldern retten muss (weil die Geldinstitute nicht abgewickelt werden können, ohne das Finanzsystem zum Einsturz zu bringen), doch unterliegen Einzelheiten unverändert streitiger Diskussion. Danach wird das Bemühen, Banken und Versicherungen aus Ihrer Mitverantwortung heraus ,zur Kasse zu bitten" und ihnen zu untersagen, mit dem Geld der Zentralbank oder der Sparer auf Währungen, Rohstoffe oder Aktien zu spekulieren, von den einen begrüßt, von den anderen hingegen als „reines Placebo“ bezeichnet. Zudem sei erkennbar, dass die handelnden Akteure sich hintereinander ,zu verstecken“ suchten, die Bundesregierung bei der Bestimmung ihres eigenen Vorgehens etwa auf ein vorangehendes europäisches Votum sowie eine greifbare Entscheidung des amerikanischen Präsidenten warte. Im Ergebnis spricht man sich heute, sicher auch mit Blick auf bevorstehende Landtagswahlen, für einen Notfallsfonds der Banken in Höhe von $€ 1 \mathrm{Mrd}$. aus, eine angesichts der vom Bankensektor ausgehenden Probleme fraglos unangemessene Größenordnung, vor allem in Anbetracht 
der bislang für die Bankenrettung aufgebrachten Kapitalhilfen in Höhe von nahezu $€ 30$ Mrd., ergänzt um jene knapp $€ 150$ Mrd. an Garantie- und Bürgschaftsleistungen, von denen nicht abzusehen ist, ob sie staatlicherseits auch tatsächlich erbracht werden müssen oder nicht.

Zudem bleibt offen, ob Vorstände und Aufsichtsräte von Aktiengesellschaften künftig länger als bisher für Fehlentscheidungen haften müssen. So wird seitens des Bundesfinanzministeriums geprüft, die bislang fünfjährige Verjährungsfrist für die aktienrechtliche Haftung im Fall von Pflichtverletzungen bei der Geschäftsführung auf zehn Jahre zu verlängern. Begründet wird dies damit, dass so Ersatzansprüche auch dann noch durchgesetzt werden können, wenn sie erst später deutlich werden. Schon länger bekannt sind demgegenüber Vorstellungen, nach denen systemrelevante Teile einer Bank künftig abgespalten werden können, um sie fortzuführen, während der verbleibende Rest abgewickelt wird. Für diese Banken soll es in Ergänzung ein Reorganisationsverfahren geben, um sie in Anlehnung an bestehende Insolvenzverfahren ggf. sanieren zu können. Für die Bankenabgabe selbst ist vorgesehen, dass sie in einen Fonds fließen sollte, der im Krisenfall die Restrukturierung oder Abwicklung von Finanzinstituten finanzieren könnte, wobei die Zahlungen je nach Engagement und Vernetzung auf den Finanzmärkten zu staffeln wären, eine für Sparkassen und Genossenschaftsbanken wichtige Differenzierung. Gegen solche Vorstellungen wenden sich erwartbar die Spitzenverbände der deutschen Wirtschaft, wobei die Bankenabgabe mit Blick auf eine mögliche Einschränkung der Kreditvergabe besonders kritisch gesehen wird; zudem bemängelt man etwaige nationale Alleingänge und offeriert als „,bessere Krisenvorsorge“ eine erweiterte Absicherung risikoreicher Geschäfte durch zusätzliches Eigenkapital.

Im Übrigen konzentrieren sich die Diskussionen auf die Besteuerung von Finanztransaktionen, das Verbot von Hedgefonds und einen multilateral vereinbarten Rahmen für Bonuszahlungen. Zumindest bei letzterem wird ein gewisses Umdenken der Angesprochenen erkennbar; dies dokumentieren das Gesetz zur Angemessenheit der Vorstandsbezüge sowie die Vorgabe, die Entlohnung am längerfristigen Erfolg einer Bank auszurichten. Auch enthalten eine Reihe von Vergütungssystemen inzwischen einen sog. Malus-Faktor, nach dem Boni zu einem Teil in einen „Topf“ fließen, der im Falle schlechterer Geschäftsergebnisse sich wieder leert. Erst nach drei bis vier Jahren stellt sich dann heraus, ob der Bonus vollständig oder nur in Teilen zu zahlen ist. Die Auflagen, nicht nur rein betriebswirtschaftliche Faktoren bei der Entlohnung zu berücksichtigen, finden dagegen nur begrenzt Eingang in die Diskussion. Hier geht es etwa um Indizes 
für die Zufriedenheit von Kunden und Mitarbeitern; auch könnten die vorgesehene Offenlegung der Vorstandsgehälter und die Erläuterung der Kriterien für die in den Konzernen gezahlten Boni durchaus versachlichend wirken. Mit der Forderung der Deutschen Schutzvereinigung für Wertpapierbesitz nach einem materiellen Einbezug der Aktionäre in Entscheidungen über Gehaltsstrukturen und Sondervergütungen anlässlich der jährlichen Hauptversammlungen wird ein weiterer Weg zu verstärkter Transparenz und Offenheit aufgezeigt. Allerdings bleiben die diskutierten Politiken bislang strikt nationalstaatlich ausgerichtet, eine internationale Initiative befindet sich lediglich , in Vorbereitung“ - dies nun freilich seit bereits zwei Jahren.

In der Zusammenfassung bleibt zu hoffen, dass veränderte und aufeinander abgestimmte Regulierungs- und Aufsichtsformen sich künftig sowohl auf nationaler wie internationaler Ebene finden. Sollte der deutliche „Schock“, der sich im Gefolge der subprime crisis für die Finanz- und Realmärkte erkennen ließ, allerdings konsequenzlos bleiben, wird man jenen Negativszenarien nähertreten müssen, die im Falle einer sich verstärkenden oder neuen Finanzkrise den Zusammenbruch der nationalen wie internationalen Finanzarchitektur voraussagen und dies mit einer möglichen Renaissance nicht-kapitalistischer Steuerungs- und Wirtschaftsformen verbinden. Alle Marktteilnehmer, Anbieter wie Nachfrager, sollten sich dessen bewusst sein und zu Reformmaßnahmen beitragen, die das erfahrene „Übermaß“ auf den Finanzmärkten eindämmen und den Primat der Politik wiederherstellen.

\section{Veränderungen der Staatlichkeit im Zeitablauf}

Schließlich mündeten die Beiträge zum ZSE-Themenheft in eine Reihe von Analysen, die sich in Vermeidung allzu aktualitätsbezogener Reaktionen der „Veränderung der Staatlichkeit im Zeitablauf" anzunehmen suchten. Sie reichen von einem Blick auf ein zeitgemäßes Verständnis der Formen und Funktionen des öffentlichen management über die Folgen des Zusammentreffens von transnationalen Rationalitäten und nationalen Traditionen bis hin zur Frage nach der Entscheidungs- und Reformfähigkeit demokratischer politischer Systeme. Die entsprechenden Ausführungen fanden zum einen eine sektorale Konzentration, so mit Blick auf die erkennbare parlamentarische Entwicklung oder die Veränderungen öffentlicher Meinung im System polyzentrischer Herrschaft, bevor zwei weiter ausgreifende Beiträge eine für die Veröffentlichung wichtige Transzendenz herstellten: einerseits durch den Blick auf die Rolle von Kirchen und Christen im demokratischen Verfassungsstaat samt des sich damit verbindenden Dis- 
kursverständnisses, das die rational vorgetragene Haltung des jeweils anderen ernst nimmt und davon absieht, Alleinvertretungsansprüche zu erheben, andererseits über die Diskussion des Staates als „Glücksmaximierer“ mit dem Ausweis der Implikationen politischen Handelns für die Determinanten menschlichen Wohlbefindens. Beide Beiträge, ganz gewiss nicht nur addenda im Rahmen der hier verfolgten Fragestellung, sind für die Zukunftsfähigkeit und die Akzeptanz des öffentlichen Handelns von beträchtlicher Bedeutung.

Jenseits der bereits aufgezeigten exogen begründeten Beeinflussungen wird man für die künftige Rolle und Funktion von „Staatlichkeit“, also des über politischadministrative Einrichtungen erbrachten öffentlichen Handelns, zunächst auf die Bedeutung der jeweiligen Rahmenbedingungen abstellen müssen. Hier machten etwa die Veröffentlichungen zum 60-jährigen Bestehen des Grundgesetzes deutlich, dass für Deutschland trotz neuer Knappheiten und eines bislang unbewältigten demographischen Wandels von einer vergleichsweise stabilen Ausgangssituation gesprochen werden kann, zumal sich das Land nicht mehr als „,klassische Einwanderungsgesellschaft“, sondern als ein Land moderner Zuwanderung versteht. Zwar ergeben sich zahlreiche neue Anforderungen, wie die nach Nachhaltigkeit oder Inklusion, doch haben sich Verfassung wie ordnungspolitischer Rahmen als eine entwicklungsfähige Basis erwiesen; sie lässt Veränderungen zu, erlaubt flexible Reaktionen und sieht, wo nötig, strukturelle Anpassungen vor. Dabei wurde im Zeitablauf allerdings auch deutlich, dass sich die positive Ausgangssituation immer dann als ,gefährdete Stabilität“ erwies, wenn das Vermögen zur Anpassung und Veränderung (mithin die Fähigkeit zur Selbstreform) nicht mehr in ausreichendem Maß gegeben war. Dies galt und gilt in Deutschland vor allem für die Ausformung des politischen Institutionenrahmens, der über die Föderalismusreformen I und II bestenfalls punktuelle Veränderungen einer als defizitär gekennzeichneten Ausgangssituation erfuhr. Hier wurde einmal mehr erkennbar, dass das bundesstaatliche Regierungs- und Verwaltungshandeln ungewöhnliche Kooperations- und Koordinationsleistungen voraussetzt, denen man über Jahrzehnte hinweg zwar cum grano salis zu entsprechen vermochte, die heute aber nachhaltigere Reformen nahe legen; sie müssten erkennbar über eine eben nur punktuell angepasste Kompetenzordnung und die Aufnahme eines Verschuldungsverbots in das Grundgesetz hinausgehen. Zudem gelten die Kooperationspotentiale innerhalb wie zwischen den Gebietskörperschaften als deutlich unausgeschöpft. Während auf Bundesebene Art. 65 GG auch weiterhin als Exkulpation dafür dient, dass es zu keinen wirklich nennenswerten Abstimmungsprozessen zwischen den „Häusern“ kommt (obwohl sich 
damit sub-optimales politisches Handeln und ein beträchtlicher Zeit- und Ressourcenverschleiß verbinden), ist aus vertikaler Sicht auf die in Haushaltsnotlagen immer schwieriger werdenden Abstimmungsprozesse zwischen Bundes-, Landes- und kommunaler Ebene zu verweisen. Während der Bund als steuerungsstarke Zentralinstanz sich von nachhaltigeren verwaltungspolitischen Konsequenzen bislang weitgehend ausnahm (sieht man von überfälligen ITbezogenen Anpassungsprozessen und einer eher technischen Entbürokratisierung ab), waren die dezentralen Gebietskörperschaften, also die Länder und der kommunale Bereich, schon aufgrund ihrer Haushaltssituation gezwungen, sich umfassenderen Reformvorhaben zu stellen. Sie folgten einer nicht nur logischen Trias von Aufgabenkritik, Funktionalreform und Strukturreform, nach der Länder wie Kommunen heute ungleich reagibler und flexibler agieren als der Bund. Dies mag die beträchtliche Ungleichzeitigkeit wie Asymmetrie zwischen den Reformvorhaben erklären, wobei auch wechselseitige Vorurteile eine Rolle spielen; die von vielen erwünschte „Reform der Reform“ im Bereich der Bildungspolitik oder auch die wenig funktional ausgerichtete Lösung der Trägerschaftsfrage nach dem SGB II (vulgo „Hartz IV“) seien als Beispiele benannt. Im letzten Fall ging man sogar so weit, einen vom Bundesverfassungsgericht als verfassungswidrig eingestuften Sachverhalt (eine sog. „Mischverwaltung“) nicht etwa durch das Abstellen eben dieses Sachverhalts, sondern durch eine Verfassungsänderung „heilen“ zu wollen. Über solche Verhaltensweisen kommt es dann zu jener verfassungspolitisch bedenklichen Praxis, nach der einzelne Artikel des Grundgesetzes (in diesem Fall Art. 91 GG) zu einer „Müllhalde“ zu werden drohen, auf die hin man ungelöste oder nur teilgelöste, in jedem Fall aber eher durch Absichtserklärungen denn materielle Politiken gekennzeichnete Probleme abzuwälzen sucht.

Gilt mangelhafte und unausgeschöpfte Kooperation und Koordination innerhalb wie zwischen den Gebietskörperschaften daher als ein zentrales Defizit des deutschen Regierungssystems, ist hinsichtlich der obersten Bundesorgane dagegen von einer in weiten Teilen stabilen Ausgangssituation zu sprechen. Hier hat man sich mit Blick auf den Bundestag, die Bundesregierung, den Bundesrat, das Bundesverfassungsgericht und schließlich den Bundespräsidenten auf punktuelle Anpassungen der jeweiligen rechtlichen und organisatorischen Grundlagen verständigt, die zu einer weiteren Stabilisierung dieser Organe beitrugen. Während der Bundestag in seinen Routinen gefestigt scheint, allerdings des „Weckrufs“ des Bundesverfassungsgerichts in der Frage weiterer, vor allem europapolitisch motivierter Souveränitätsabgaben bedurfte, steht die Bundesregierung trotz eines 
letztlich stabilen Leistungsrahmens in der Gefahr, zunehmend reaktiv und damit nur noch situativ zu verfahren. Die erkennbaren Schwierigkeiten der seit Oktober des vergangenen Jahres amtierenden Koalition, strittige politische Fragen innerhalb vertretbarer Zeiträume zu beantworten und den vom Souverän übertragenen Gestaltungsauftrag auch wahrzunehmen, sind primär selbstverschuldet. Dies gilt vor allem für eine handwerklich erkennbar unzureichende Koalitionsvereinbarung, die in dieser Intensität ungewöhnliche Fixierung auf einen anstehenden Landtagswahltermin (die die materielle Gesetzgebungstätigkeit fast zum Erliegen brachte) sowie ein politisches Führungsverhalten, das der Moderationsrolle im Rahmen einer Großen Koalition, nicht aber der gegenwärtigen soliden Mehrheitskonstellation angemessen erscheint.

Der Bundesrat sucht demgegenüber nach den durch die Arbeit der Föderalismuskommissionen ermöglichten (punktuellen) Reformen zu einem neuen Selbstverständnis zu finden, kämpft aber in sich um eine verstärkte Kohärenz. So ist es dem Bund noch immer ein vergleichsweise Leichtes, bei strittigen Fragen über die Parteigrenzen hinweg Mehrheiten ,einzukaufen“. Dies wird solange der Fall sein - und damit latent die bundesstaatliche Ordnung beschädigen -, als die Betroffenen territorial, demographisch, ökonomisch und soziokulturell sehr unterschiedlich geprägte Länder repräsentieren. Die angestrebte „Einheit in der Vielfalt" verbleibt im Alltag des deutschen Föderalismus doch meist eher auf dem Papier; hier durch ein weiteres Insistieren auf der föderalstaatlichen Solidarfunktion und eine Wiederbelebung der Frage nach einer Länderneugliederung motivierend zu wirken, erscheint zumindest mittelfristig angezeigt.

Das Bundesverfassungsgericht schließlich steht zunehmend in der Gefahr, zu einer Einrichtung politischer Letztentscheidungen zu werden. Der Trend, politisch ungelöste Konflikte dem Gericht zuzuweisen oder eine Niederlage auf parlamentarischer Ebene durch Anrufung des Gerichtes zu mildern, hat sich deutlich verstärkt und droht im letztbenannten Fall missbräuchlich zu werden. Dabei wird man dem Gericht das Kompliment machen können, dass es trotz einzelner strittiger Urteile meist zu einer dem Grundrechtsschutz und der Stabilität der bundesstaatlichen Ordnung angemessenen Spruchpraxis fand. Dass sich damit künftig auch wieder eine gewisse öffentliche Zurückhaltung (also jenes immer wieder angeführte judicial restraint) anbietet, sei in Erinnerung gerufen.

So verbleibt beim Blick auf die obersten Bundesorgane das Amt des Bundespräsidenten, das unter dem derzeitigen Amtsinhaber eine weitere Stabilisierung, in Teilen aber auch ungewöhnlich kritische Diskussion erfährt. Letzteres bezieht sich auf eines jener wenigen materiell wirkungsvollen Instrumente, über die das 
Staatsoberhaupt verfügt: die öffentliche Stellungnahme in Reden und Schriften. Hier vergleicht man den Präsidenten kritisch mit seinen Vorgängern und sieht ihn in krisenhafter Zeit als zu wenig aktiv und inspirierend. Gerade aufgrund seiner ökonomischen Primärqualifikation würde es ihm in der Tat wohl gut anstehen, auf die mit der Finanz- und Wirtschaftskrise verbundenen Herausforderungen hinzuweisen und schnellere als die bislang erkennbaren Reaktionen einzufordern.

"Gefährdete Stabilität“ bleibt mithin eine Metapher, die die Veränderung der Staatlichkeit im Zeitablauf adäquat zu kennzeichnen scheint. Sie mahnt weitere institutionelle Reformen und ein flexibleres, proaktiveres Handeln der politischen Einrichtungen und ihrer Akteure an - wohlwissend, dass ein solches Handeln voraussetzungsvoll ist: Da Demokratie sich eher selten jene Rahmenbedingungen schafft, auf denen sie basiert, ist ein Grundkonsens über die Rolle und Funktion von „Staatlichkeit“ unabdingbar. Ein erinnernder Blick auf die gesellschaftlich-historische Entwicklung des Staatsbegriffs und seiner Teleologie mag in diesem Zusammenhang darauf verweisen, dass ein entsprechender Konsens ideengeschichtlich eher die Ausnahme denn die Regel darstellt und folglich im Rahmen staatswissenschaftlicher wie staatspraktischer Analysen stets berücksichtigt werden sollte.

\section{Illusion der Stabilität: Der Staat als Politikum}

Theorien des Staates und seiner Funktionen wurden in Deutschland traditionell nicht nur in jenen Wissenschaften entworfen, für die sich seit dem späten 18. Jahrhundert der Begriff der Staatwissenschaften durchsetzte. Zwar hatten am deutschen Staatsdiskurs Juristen, vor allem die sog. Staatsrechtslehrer, immer einen hohen Anteil, doch wurde seit der Frühen Neuzeit auch in vielen anderen Disziplinen gelehrte akademische Staatsdeutung betrieben. Eine besonders wichtige Rolle kam dabei der Philosophie zu, aber auch den konfessionellen Theologien, weil hier wie dort Ethik als relativ autonome Teildisziplin institutionalisiert war; im Rahmen der Ethik als normativ orientierter Leitdisziplin zur Deutung aller Basiseinrichtungen des menschlichen Weltumgangs gewannen unausweichlich die Politische Ethik und hier speziell die Staatsethik eigene hohe Bedeutung. Mit dem Auseinandertreten von Staat und bürgerlicher Gesellschaft, wie es sowohl in der klassischen schottischen Ethik und Ökonomie, besonders folgenreich bekanntlich bei Adam Smith, als auch im deutschen theologischen und philosophischen Diskurs um 1800, begrifflich besonders prägnant bei Schleiermacher und Hegel, analysiert wurde, wurden dann überkommene Deutungsmuster des 
Staates und seiner Funktionen zunehmend von normativ orientierten Analysen des Verhältnisses von Staat und Gesellschaft abgelöst. Galt dabei die bürgerliche Gesellschaft als ein Ort von Markt, Austausch, Konkurrenz und legitimem Konflikt, so wurde der Staat vorrangig als jene Ordnungsmacht gedacht, die die bürgerliche Gesellschaft durch Recht und starke Institutionen der Durchsetzung des Rechtsgehorsams pazifiziert. Die Frage nach der inneren Einheit des Gemeinwesens, nach seiner Integration, gewann dabei besondere Bedeutung.

In idealtypischer Vereinfachung hoher argumentativer und positionspolitischer Komplexität lassen sich für den deutschen Diskus zwei miteinander konkurrierende Antworten unterscheiden. An sie zu erinnern kann in der gegenwärtigen Diskussion über „die Wiederkehr des Staates“ und mit Blick auf die vielfältigen Krisenphänomene zentraler gesellschaftlicher Organisationen und Institutionen hilfreich sein, um zu erkennen, dass derzeit im Kern keine prinzipiell neuen Fragen diskutiert werden, sondern angesichts eines zweifelsohne verstärkten Problemdrucks und vielfältig veränderter kultureller Bedingungen nur sehr alte Fragen nach dem inneren Zusammenhalt des "Gemeinwesens“ wieder auf die politische Agenda zurückgekehrt sind. Auch wenn nun ganz andere politische und ökonomische Rahmenbedingungen - insbesondere die Prozesse weiterer europäischer Integration und kapitalistischer Globalisierung - sowie grundlegend neue kulturelle Voraussetzungen des Politischen - weltweite Migration, neue Kommunikationstechnologien und eine radikale Vielfalt höchst unterschiedlicher Lebensstile und Lebensentwürfe - zu berücksichtigen sind, bleibt doch die alte Frage nach den gesellschaftlichen wie kulturellen Voraussetzungen effektiven Staatshandelns aktuell.

Die Diskussion politisch relevanter öffentlicher Moral und innerer Einheit des Gemeinwesens gehören zu den klassischen Themen politischer Ethik. Idealtypisch lassen sich für den deutschen Diskurs zwei Antworten unterscheiden: die von den konsequent liberal denkenden Kantianern verfochtene Integration des politischen Verbandes allein durch Recht und die von den christlichen Konservativen sowie von vielen Sozialdemokraten verfochtene Integration durch überindividuell verbindliche Kulturwerte. Natürlich gab es und gibt es im deutschen politischen Denken zwischen diesen beiden Konzepten vielfältige Positionen der Vermittlung, schon seit dem frühen 19. Jahrhundert. Aber zur präziseren Bestimmung der gegenwärtigen Herausforderungen von Staatlichkeit dürfte es hilfreich sein, zunächst die gegensätzlichen Modelle zu skizzieren, um dann ihre je eigene Leistungskraft sowie ihre je besonderen Schwächen zu bezeichnen. 


\section{Das liberale Modell: Immanuel Kant und das Grundgesetz}

Das liberale Modell des Politischen ist mit hoher begrifflicher Prägnanz von Immanuel Kant entwickelt worden. Es hat im deutschen akademischen Staatsdiskurs allerdings seit dem frühen 19. Jahrhundert eine deutlich geringere Prägekraft entfaltet als die organologischen Staatstheorien der Romantiker und die Staatsmetaphysik im sog. „deutschen Idealismus“. Zwar haben Kulturtheoretiker wie Max Weber, seiner Ausbildung nach Jurist, und kluge metaphysikkritische Weimarer Staatsrechtslehrer, wie insbesondere Hans Kelsen, den entscheidenden Kantischen Impuls aufgenommen, sowohl um der Freiheit der Bürger als auch um der Effizienz des Staates willen von ihm nicht allzu hoch zu denken, also den Staat ohne metaphysischen Ewigkeitsglanz zu entwerfen. Aber erst im Staat des Grundgesetzes wurde in Philosophie, Jurisprudenz und auch protestantischer Theologie die spezifische Leistungskraft der Kantischen Politischen Ethik erkannt. Sie lässt sich in gebotener Kürze so skizzieren: Um der menschenrechtlich garantierten, insoweit prinzipiell vorstaatlichen Freiheit der Einzelnen willen wird der Staat auf die äußere Rechtssphäre beschränkt. Der freiheitliche Rechtsstaat ist religiös oder weltanschaulich radikal neutral und darf sich deshalb nicht als eine moralische Anstalt, als ein Sittenstaat oder aktiver Sinnstifter verstehen. So wenig er seinen Bürgern irgendeinen religiösen Glauben vorschreiben darf, so wenig darf er Gesinnungskonformität oder Zivilreligion einklagen. Für eine freie Gesellschaft sei die strikte Unterscheidung von innen und außen, privat und öffentlich, Legitimität und Legalität sowie die institutionell gesicherte Differenzierung von Politischem und Religiösem konstitutiv. Die offene Gesellschaft freier Bürgerinnen und Bürger dürfe deshalb nicht durch eine für gemeinschaftlich erklärte, folgerichtig dann als gemeinverbindlich behauptete Idee des Guten integriert werden. Allein das formale Recht verbinde freie Subjekte zum freiheitlichen politischen, demokratischen Verband. Recht wird in der Kantischen Tradition mit großer gedanklicher Stringenz als ein rein formales Regelsystem gedeutet und der Staat, im Gegenüber zu den Kirchen, Synagogengemeinden und sonstigen moralischen Sinnstiftungsagenturen, auf die Fiktion konsequenter weltanschaulicher Neutralität verpflichtet - auch wenn seine Repräsentanten, wie sich immer wieder zeigen lässt, in ihrem politischen Handeln und auch in ihren Rechtsakten von je besonderen kulturellen oder religiösen Prägungen und weltanschaulichen Gewissheiten nicht völlig frei sind. Für die Kantische Tradition des deutschen Staatsdenkens gilt: Der Staat soll keine Werte predigen oder Sinn stiften wollen, sondern sich in freiheitsdienlicher Selbstbeschränkung damit begnügen, die äußeren Freiheitssphären der Bürger voneinander abzugrenzen 
und den Rechtsfrieden zu garantieren. In dieser prägnanten Selbstbegrenzung liegt die Stärke des liberalen Staates. Seine selbst gewollte konstitutive Schwäche, oder anders formuliert: sein Metaphysikverzicht macht seine grandiose Stärke aus.

Der moralminimalistische Rechtsstaat will die Gesellschaft, den Ort von Tausch, Konkurrenz und Kommunikation der freien Subjekte, nur mit einem relativ lockeren Integrationsband, dem Recht, überziehen, bloß den unverzichtbaren „Ordnungsrahmen“ dafür bilden, dass Gesellschaft funktionieren kann. Machtphantasien der starken Steuerung gesellschaftlicher Tauschprozesse und Interessenkonflikte liegen diesem Staat fern. Er will, dass es in der Gesellschaft, bei aller legitimen Vielfalt, Konkurrenz und Interessengegensätzlichkeit, zivil, also rechtlich geordnet zugeht. Aber er maßt sich nicht an, in den innergesellschaftlichen Auseinandersetzungen für einen der partikularen Akteure, etwa ihre je besonderen Interessen oder ihre individuelle Weltsicht, Partei zu ergreifen. Er hält sich hier vornehm zurück. Und daran tut er gut. Er weiß in seiner ,formalistischen" Bescheidenheit und antimetaphysischen Selbstbegrenzung um die Grenzen seines Wissenkönnens. Der Staat könnte nur dann mehr als die Gesellschaft wissen, wenn er an gleichsam metaempirischer göttlicher omniscientia, dem Allwissen eines das Ganze der Wirklichkeit und die Fülle der Zeiten wahrnehmenden Beobachtergottes jenseits aller (unausweichlich relativierenden) Perspektivität teilhaben könnte - wie auch immer. Aber er weiß, dass er dies nicht kann, und deshalb weiß er auch, dass er niemals weiter blicken kann als die Gesellschaft oder genauer: der wahrnehmungssensibelste, jeweils blickstärkste Akteur in der Gesellschaft. Der konsequent liberale Staat ist deshalb immer darauf angewiesen, sich das in der Gesellschaft von ganz unterschiedlichen Akteuren - Wissenschaftlern, Wirtschaftseliten, Intellektuellen etc. - produzierte Wissen zu eigen zu machen - nicht um allemal illusionärer Steuerung willen, sondern im Interesse, den „Rahmen“, den er auf der Grundlage der besonders fest (aber nicht „ewig“) definierten Basis der Verfassung immer neu zu entwerfen hat, um gesellschaftlicher Produktivität willen realitätsnah zu konstruieren. Die bisweilen zu lesende Behauptung, dieser konsequent liberale Staat sei deshalb ethisch defizitär und gleichsam morallos gedacht, ist allerdings falsch. Indem er darauf verzichtet, selbst als Sinnstifter oder moralischer Akteur aufzutreten, muss er von den Bürgern unausweichlich eine spezifische Bürgertugend erwarten: die entschiedene Bereitschaft, sich im individuellen Verhalten the rule of law zu eigen zu machen. In ethischer Hinsicht ist von den Bürgern einschließlich der Inhaber politischer Ämter nur eine Tugend zu fordern: die Bereitschaft, 
strikten Rechtsgehorsam zu internalisieren. Andere Bürgertugenden mag man im Interesse einer zivilen politischen Kultur wünschen und erhoffen. Aber der Staat darf sie nicht einklagen wollen, um der vorstaatlichen Freiheit der Bürger und um seiner eigenen Funktionsfähigkeit willen.

\section{Der christlich-konservative Gegenentwurf}

Gegen Kants Rechtsphilosophie wurden schon in den 1790er Jahren und verstärkt dann seit 1800 Einwände formuliert, die die politischen und speziell staatstheoretischen Diskurse bis in die zyklisch wiederkehrenden Werte- oder „Grundwerte“-Debatten des Bundesrepublik hinein bestimmten. Noch immer fehlen diskurshistorische Studien über die Kantrezeption im ausgehenden 18. und frühen 19. Jahrhundert. Deutlich ist bisher nur, dass Kant in protestantischen Theologischen Fakultäten deutlich früher als in Juristischen Fakultäten gelesen wurde. Klare disziplinäre Grenzen zwischen Theologie und Philosophie gab es damals insoweit nicht, als die Mehrzahl der Inhaber philosophischer Professuren auch Theologie studiert hatten und durchaus wieder in Theologische Fakultäten zurückkehren wollten, aus ökonomischen Gründen, waren hier Lehrstühle doch deutlich höher dotiert, die Hörerzahlen (mit entsprechendem Hörergeld) deutlich größer und sehr häufig mit lukrativen kirchlichen Pfründen - freien Wohnungen, Einkommen aus Grundbesitz etc. - bei vergleichsweise geringen kirchenbezogenen Aufgaben, etwa der Pflicht, einmal im Monat zu predigen, verbunden. In diesem sehr intensiven theologischen Kant-Diskurs wurden sowohl die spezifische Leistungskraft als auch die möglichen Schwächen seiner Konzeption früh schon kontrovers diskutiert. Als entscheidend erwiesen sich hier zunächst die Argumente vieler entschieden christlicher frühkonservativer Kant-Kritiker.

Diese Theoretiker des frühen Konservativismus hatten gegen den als abstrakt erlittenen „Formalismus“ der Kantischen praktischen Philosophie aristotelische Denkfiguren reformuliert und wieder substantielle Gemeinschaftswerte geltend gemacht. Ihr entscheidendes Argument lautete: Eine bloß äußerliche Integration durch das formale Recht leiste nur einer brutalen Konkurrenz- und Ellenbogengesellschaft Vorschub, in der sich die Stärkeren erbarmungslos über die Schwächeren, auf Solidarität und Gemeinsinn angewiesenen Hilfsbedürftigen hinwegsetzten. Das freie Individuum der Liberalen deuteten sie mit den Sprachmustern der alten christlichen Sündenlehre als egozentrischen und um sich selbst kreisenden Sünder, der in der Fixierung auf seine unmittelbaren Interessen weder Gemeinsinn noch sonstige Solidartugenden entwickeln könne. In diesen Debatten über „den Einzelnen“ und „die Gemeinschaft“ waren die Morallasten von vorn- 
herein zugunsten „der Gemeinschaft“, „,des größeren Ganzen“, „des Sozialen“ und des „Gemeinwohls“ - wohl der ideologieanfälligste Begriff der modernen politischen Sprache - verteilt. Ein protestantischer Konservativer, wie etwa Friedrich Julius Stahl, und frühe Theoretiker der dezidiert restaurativen katholischen Soziallehre, wie etwa Adam Müller und Franz von Baader, betonten Naturrechtsüberlieferungen, nach denen niemand rein aus sich und für sich lebe. Jeder Mensch sei von Geburt an immer schon eingebunden in bergende sittliche Gemeinschaften, wie insbesondere die Familie. Stark geprägt durch Traditionen der lutherischen Ständeethik und der überkommenen römisch-katholischen Naturrechtstradition insistierten die christlichen Konservativen des vormärzlichen Deutschland darauf - nicht selten mit Rekurs auf Hegels etatismusfreundliche Hochschätzung des Staates als Inkarnation des „objektiven Geistes“ -, dass die Bürger in mehr als nur äußerlichen, rechtlichen Beziehungen zueinander stehen müssten, solle die moderne bürgerliche Gesellschaft nicht zu einem brutalen Kampfplatz von Egoisten pervertieren und humane Solidarität mit den Schwächeren verloren gehen. Über die nur äußere Verbindung der Bürger hinaus klagten sie deshalb auch eine innere Vergemeinschaftung ein: die Bindung an eine gemeinsame Idee des Guten, an verbindliche Normen und Kulturwerte, an überkommene sittliche Traditionen. Nur eine solche vorrechtliche, sittliche Einheit könne verhindern, dass die Gesellschaft durch Interessenkonflikte und Gruppenkämpfe zersetzt und die gewachsene Ordnung durch eine verabsolutierte Freiheit der Einzelnen zerstört werde.

In der Perspektive der christlich Konservativen ist der Staat ein sozialer Sittenstaat, der über äußeren Rechtsgehorsam hinaus auch innere Loyalität, eine zivilreligiöse Gesinnungstreue und Solidarität mit den Schwächeren beanspruchen soll. Deshalb sprachen sie in sehr hohen Tönen gern vom Kulturstaat oder vom Sozialstaat, beschworen das bonum commune bzw. Gemeinwohl und verstanden ihre christlichen Parteien als Weltanschauungsparteien und religiös-sittliche Gesinnungsgemeinschaften. Seit der Formierung des deutschen politischen Katholizismus in der Revolution von 1848/49 und der Gründung konservativer Parteien, in denen Lutheraner das Sagen hatten, und seit der Gründung der Zentrumspartei, der Partei des katholischen Milieus, pflegen die christlichen Konservativen eine Werterhetorik, in der altehrwürdige Begriffe des Neuen Testaments, etwa der Begriff der Nächstenliebe, zur Rechtfertigung starker staatlicher Institutionen diente, mit denen die Freiheitsräume der Individuen begrenzt werden sollten. Ihre Parteiprogramme ließen immer eine Tendenz zur Übermoralisierung des Politischen erkennen. Dies zeigte sich zunächst in den Visionen eines 
„christlichen Staates“, in dem politische Institutionen mit den Kirchen zum Zwecke der Versittlichung der Gesellschaft eng kooperieren.

Im frühen 19. Jahrhundert wurden die „Kulturwerte“ zunächst durch christliche Sprache legitimiert. Als die hohen sozialen Kosten der industriellen Modernisierung sichtbar wurden und sich die deutsche Gesellschaft in antagonistische Klassen und heterogene sozialmoralische Milieus ausdifferenzierte, gewann auch das Moralische eine plurale Gestalt. Die deutschen Parteien konkurrierten nicht nur um die politische Macht, sondern wollten jeweils auch ihre Weltanschauung durchsetzen. Neben den christlich Konservativen und den Sozialdemokraten, die ihre Grundwerte zunächst bei Marx und Engels fanden, setzten auch die liberalen Parteien im Kaiserreich zunehmend auf Werteproduktion. Über „Werte“ suchte man die eigene Klientel an sich zu binden, und zugleich konnte man die harten Kämpfe um politische Macht und Einfluss als Veranstaltungen inszenieren, die primär um der wahren Gemeinschaftswerte willen zu führen seien.

\section{Staatsfunktionen und Zeitgeist}

Die Dauerdiskussionen um Bürgerfreiheit, Grundkonsens und politische Moral bewegen sich bis heute im Spannungsfeld von formaler Rechtsintegration und materialer Wertintegration. Seit den amerikanischen Kontroversen zwischen Neoliberalen und Kommunitaristen stehen mögliche Vorzüge und Schwächen beider Modelle im Vordergrund der politisch-ethischen Debatte. Kann die parlamentarische Demokratie allein durch Legalität und Verfahrenslegitimität funktionieren? Bedarf sie nicht auch einer moralischen Grundhaltung der Bürger, etwa Tugenden wie Gemeinsinn und elementarer Solidarität mit dem schwachen anderen? Ein freiheitlicher Staat darf solche „moralische software“ nicht bilden wollen. Ein Tugendstaat wird schnell zur Hölle des zivilreligiösen Gesinnungsterrors, kann man seit Hölderlin wissen: „Beim Himmel! Der weiß nicht, was er sündigt, der den Staat zur Sittenschule machen will. Immerhin hat das den Staat zur Hölle gemacht, dass ihn der Mensch zu seinem Himmel machen wollte.“ Aber dies bedeutet nicht, dass der liberale Staat ohne Bürgertugend zu funktionieren vermag. Die entscheidende Frage an konsequent liberale Theoretiker der Integration allein durch Recht lautet deshalb: Wie und wo sollen jene Bürgertugenden gebildet werden, die allererst die Bereitschaft zum Rechtsgehorsam ermöglichen und zur Stärkung von Gemeinsinn beitragen?

Demgegenüber lautet die entscheidende Frage an die Wertintegrationstheoretiker: Wie soll in einer modernen, posttraditionalen Gesellschaft, die von einem 
breiten ethischen Pluralismus und der Koexistenz gegensätzlicher religiöser und ethisch-weltanschaulicher Grundhaltungen geprägt ist, politische Integration durch Werte erfolgen können? Ist es nicht illusionär, angesichts des geschichtlich nun einmal gegebenen Pluralismus der religiösen Grundhaltungen, moralischen Optionen und individuellen Lebensentwürfe noch auf allgemein verbindliche Werte zu setzen? Sind nicht auch die sog. „Grundwerte“ bloß partikulare Wertorientierungen, die einzelne Gruppen für allgemeinverbindlich erklären? Versucht nicht jede Weltanschauungsgemeinschaft, ihre besonderen Wertorientierungen zu verallgemeinern? Wenn dies der Fall ist, dann droht Integration über Werte politisch destruktiv zu werden und Desintegration noch zu verschärfen. Wer bloß seine besonderen Wertorientierungen politisch allgemeinverbindlich machen will, verschärft nur die permanenten Wertkonflikte in pluralistischen Gesellschaften. Sind Wertintegrationsprogramme also politisch kontraproduktiv?

Fragen dieser Art gewinnen an Gewicht, sieht man die bei vielen Bürgern in der Bundesrepublik zu beobachtende wachsende Skepsis gegenüber großen, pathetisch verkündeten Wertbegriffen. Wer etwa ein „christliches Menschenbild“ oder „freiheitliche Werte“ beschwört, droht nur reale Verschiedenheit durch hypostasierte Einheitsbegriffe abzublenden. In einer historischen Perspektive lässt sich beobachten: Je fragmentierter und konfliktreicher moderne Gesellschaften sind, desto lauter wird nach Werten und neuer Moral gerufen. Neue Werte und mehr Gemeinsinn haben in den letzten Jahren keineswegs nur konservative Intellektuelle oder Politiker von CDU und CSU gefordert. In den aktuellen Diskussionen um das Verhältnis von Staat und Zivilgesellschaft sowie in den Debatten um globalisierte Finanzmärkte und die Weltfinanzkrise haben sich auch viele Sozialdemokraten, Grüne und Liberale die Leitbegriffe der Kommunitaristen zu eigen gemacht und in stark moralisierender Sprache die Krise auf die mangelnde ethische Verantwortung und asoziale Unmoral der Banker, ihre Habgier, zurückgeführt. Viele politische Akteure und Intellektuelle, die in den sechziger und siebziger Jahren in pathetischer Emanzipationssprache mehr Selbstverwirklichung einklagten, treten in den Feuilletons nun für die Wiederkehr der Tugend ein. Manche rufen nach einem neuen preußischen Ethos, um den Standort Deutschland zu stärken, andere fahnden nach jenen sozialmoralischen Ressourcen, die den Gemeinsinn stärken sollen, und wieder andere beschwören mit Blick auf die vielfältigen Korruptionsskandale in der Republik neue Dekaloge. An moralischen Pathosformeln herrscht in der Berliner Republik wahrlich kein Mangel. Aber Werte fallen nicht vom Himmel, und Moral entsteht nicht dadurch, dass man nach ihr ruft. 
Integrationsprozesse scheinen in posttraditionalen Gesellschaften einem rapiden sozialen Strukturwandel zu unterliegen. Die großen, zentralistischen Integrationsmuster verlieren zunehmend an Bedeutung. Staatliches Recht stiftet häufig keinen sozialen Frieden mehr, und Bürokratien, für Max Weber noch die Garanten zweckrational effizienten öffentlichen Handelns, produzieren nicht selten, wie man gerade am Beispiel der Bildungs- und Wissenschaftspolitik sehen kann, dysfunktionale, insoweit irrationale Entscheidungen, deren desaströse Folgewirkungen sich kaum noch beheben lassen. Oft lassen sich demokratisch legitimiertes Recht und gesellschaftliche Vorstellungen von Gerechtigkeit gar nicht mehr aufeinander beziehen, was zur Schwächung des Rechts beiträgt.

Angesichts der globalen Herausforderungen von Klimawandel, Kampf gegen den Terrorismus, Friedenssicherung und Nord-Süd-Problematik scheinen auch die Möglichkeiten zu schwinden, politischen Konsens in den überkommenen Formen demokratischer Willensbildung bzw. über Verfahrenslegitimität zu erzeugen. Die Muster politischer Partizipation haben sich in den vergangenen Jahrzehnten deutlich gewandelt. Die Mitgliederpartei, in der man sich für alle möglichen Themen an ein politisches Milieu bindet, kann in einer Kultur der vielfältig fragmentierten patchwork-Identitäten nur noch kleine Gruppen integrieren. Politische Partizipation wird zum ,widerspruchsvollen Vielengagement“ (Ulrich Beck): Die konfessorische Bindung an eine Partei wird bei einer wachsenden Zahl von Menschen abgelöst von zeitlich überschaubaren, thematisch konkreten und vorrangig lokalen politischen Engagements in Bürgerinitiativen, Netzwerkgruppen oder auch Kirchengemeinden. Diese Gruppen erzeugen in der Konkurrenz zu Parteien und Verbänden vielfältige Konflikte. Sie tendieren, wie der neue Öko-Pietismus zeigt, zur Übermoralisierung des Politischen und wirken so polarisierend. Aber sie stellen auch kleine Konsensinseln dar, Orte, an denen thematisch begrenzte Teilkonsense ausgehandelt werden. Insoweit lassen sie sich als Kräfte partieller Homogenisierung deuten. Viele Sozialwissenschaftler vermuten, dass solche kleinen ,zivilgesellschaftlichen“ Integrationsmuster, die intermediären Stadien von wechselseitiger Abstimmung und Einheitsbildung, zukünftig noch an Bedeutung gewinnen werden. Zentrale politische Integration, die Entscheidungsproduktion in den klassischen demokratischen Einrichtungen, wird dadurch aber keineswegs überflüssig, auch wenn dies in den institutionentheoretisch wenig prägnanten governance-Diskursen gern suggeriert wird. Denn der in den heterogenen „Netzwerken“ neuer sozialer Bewegungen oder durch transnational agierende NGOs erzeugte politische Druck macht Koordination, Steuerung und Entscheidungsproduktion nicht obsolet, sondern dürfte, 
genau umgekehrt, einen höheren Bedarf an zentraler Koordination und Steuerung erzeugen. Weil zivilgesellschaftliche Integration in intermediären Gruppen Integration über das politische Institutionensystem ergänzen, aber nicht ersetzen kann, sind diese Gruppen immer auch danach zu fragen, wie sie es mit Regelkonsens und Mehrheitsregel halten.

Auf steigende soziale Differenzierung, moralischen Pluralismus, neue religiöse Vielfalt und postmoderne Pluralität selbstbestimmter individualistischer Lebensstile reagieren Gruppen der Gesellschaft mit dem Rückzug in homogene Ghettos und autoritäre Fundamentalismen. Je unübersichtlicher die soziale Welt wird, desto stärker klammern sich viele Menschen an neue Autoritäten und einfache Wahrheiten. Solche Fundamentalismen haben seit den siebziger Jahren in allen westlichen Gesellschaften an Einfluss gewonnen. Sie stellen zunehmend auch eine gewichtige ethische Herausforderung für die großen Volkskirchen dar.

Wer die offene Gesellschaft mündiger Bürger und Bürgerinnen verteidigen will, muss sich mit diesen Fundamentalismen auseinandersetzen. Solche Auseinandersetzung ist konfliktträchtig. Denn sie konfrontiert mit Fragen, für die es in den überkommenen Modellen der Definition des liberal-parlamentarischen Rechtsstaates keine zureichenden Antworten gibt. Wie wollen wir auf Dauer mit religiös-weltanschaulichen Akteuren politisch umgehen, die aufgrund ihrer Wertorientierungen konsequent die freiheitsdienliche Differenzierung von Recht und Moral, Politik und Religion verweigern? Dies gilt besonders für solche fundamentalistischen islamischen Gruppen in unserer Gesellschaft, die zentrale Elemente ihrer Identität aus einer prinzipiellen Kritik „des Westens“ gewinnen, aber für ihre Angehörigen gleichwohl ein Recht auf Einbürgerung beanspruchen. Sollen auch solchen Einwanderern aus muslimischen Ländern Staatsbürgerrechte zuerkannt werden, die eine neue Einheit von Religion und Politik fordern sowie die Idee von Menschenwürde und vorstaatlicher Freiheit des Einzelnen aus religiösen Gründen bekämpfen? Dabei geht es um einzelne, in Deutschland bisher marginale muslimische Gruppierungen, nicht um den Islam, den es so wenig wie das Christentum gibt. Bessere Integration lässt sich langfristig nur erreichen, wenn die politisch Verantwortlichen die Ressourcen für erheblich gesteigerte Bildungsprogramme zur Verfügung zu stellen bereit sind. Noch immer weiß die deutsche Öffentlichkeit nur relativ wenig über die häufig sehr geschlossenen Lebenswelten islamischer Bürger und ihre besonderen Wertorientierungen. Bildungsdefizite aber verstärken Ängste und diffuse Vorurteile. Mehr Verschiedenheit bedeutet in aller Regel auch: mehr Konflikt. Desto wichtiger ist die Stärkung 
der demokratischen Einrichtungen, in denen Konflikte nach geordneten Verfahren zu bewältigen versucht werden.

Seit dem späten 18. Jahrhundert war gesellschaftliche Modernisierung immer von „Sittenverfall“ begleitet. Überkommene moralische Selbstverständlichkeiten wurden kritisch negiert oder verloren an lebensdienlicher Plausibilität. Wo es keine Stände mehr gibt, lassen sich auch keine Standesethiken bewahren, und die vielen „Leitbilder“ und Ethik-Codices, mit denen sich viele Firmen und Organisationen heute eine moralische corporate identity zu geben versuchen, sind nur ein sehr schwacher Ersatz für jene substantielle Sittlichkeit, die traditionell durch religiösen Glauben erzeugt oder zumindest gestärkt wurde. Tugend und Moral sind in posttraditionalen komplexen Marktgesellschaften deshalb notorisch knappe Güter. Dies ist politisch langfristig prekär. Denn den Wissensgesellschaften eignet ein bisher ungekanntes $\mathrm{Ma} \beta$ an Selbstgefährdungspotentialen. Sie benötigen gerade in den Funktionseliten hohe moralische Selbstreflexionskapazität. Je größer die Handlungsmöglichkeiten, desto größer ist auch der Bedarf an moralischer Selbstbegrenzung des Menschen. Doch solche Moral wird durch fortschreitende Modernisierung permanent zersetzt.

\section{Exkurs: Kirchen als Moralproduzenten im demokratisch-liberalen Staat?}

Gerade demokratisch verfasste offene Gesellschaften benötigen deshalb Organisationen und Institutionen, in denen dem fortwährenden Moralverzehr entgegengewirkt wird. Als solche Institutionen der Erinnerung an moralische Überlieferungen und der damit verknüpften Akkumulation moralrelevanter Ressourcen galten im deutschen Diskurs über Staat und Gesellschaft traditionell vor allem die Kirchen und sonstigen religiösen Gemeinschaften. Man schrieb ihnen ein moralisches Mandat zu, das sie sehr gern auch wahrnahmen, und deutete die religiöse Vergemeinschaftung in Kirchen und Glaubensgemeinden trotz aller tiefen Differenzen und Spannungen zwischen Protestanten und Katholiken letztlich als eine sozialintegrative Leistung. Gerade den beiden großen Volkskirchen wurde deshalb immer attestiert, unverzichtbare vorpolitische Ligaturen in der pluralistischen Gesellschaft zu bilden. Man sah sie als moral communities, denen dank der ritualisierten kollektiven Erinnerung an ihre je besondere religiöse Überlieferung und moralische Tradition die Funktion zukam, genau jene Bürgertugenden zu bilden, die die Erosion der offenen Gesellschaft zum bloßen Kampfplatz rücksichtsloser Individuen verhindern. Der berühmte Böckenförde-Satz von den sozialmoralischen Voraussetzungen des freiheitlichen Staates, die dieser selbst nicht garantieren könne, ist im bundesdeutschen Diskurs von Politikern 
wie von Kirchenvertretern so oft wiederholt worden, dass er große suggestive Überzeugungskraft gewann. Eine entscheidende Frage wurde darüber vergessen: Sind religiöse Akteure, wie insbesondere die beiden großen Volkskirchen, denn tatsächlich dazu imstande, ihre religiösen Symbolbestände so zu pflegen, dass die politische Verantwortungsbereitschaft der Bürger, ihr Gemeinsinn und ihre Bereitschaft zu Rechtsgehorsam gestärkt wird?

Der im politischen Diskurs übliche Hinweis darauf, dass knapp zwei Drittel aller Deutschen den beiden großen Kirchen angehören, kann nicht über die vielen Krisenphänomene in den Kirchen selbst hinwegtäuschen. Ihre geistliche wie moralische Strahlkraft hat in den letzten Jahren dramatisch abgenommen, und das intellektuelle sowie theologische Niveau der in den Kirchen geführten Debatten lädt Nachdenkliche, argumentativ Orientierte - welcher weltanschaulichen Herkunft auch immer - nicht dazu ein, hier engagiert mitzustreiten. Das peinlich schlechte Krisenmanagement, das führende Kirchenvertreter in der Missbrauchskrise besonders der römisch-katholischen Kirche gezeigt haben, lässt einen erheblichen Mangel an Professionalität in den kirchlichen Funktionseliten erkennen. Eine relevante sozialwissenschaftliche Forschung zu den Kirchen gibt es in der Bundesrepublik nicht, und so lässt sich die Frage, ob die Kirchen denn zu leisten vermögen, was von ihnen politisch erwartet wird, nicht auf Grundlage methodisch seriös erhobener Daten beantworten. Deutlich ist jedoch, dass beide großen Kirchen seit Jahren erodieren und sich insbesondere die römischkatholische Kirche sehr schwer damit tut, elementare Prinzipien der parlamentarischen Demokratie und diskursive Spielregeln der offenen Gesellschaft anzuerkennen. Dass von ihnen eine Stärkung der Demokratie zu erwarten ist, kann man aus guten Gründen bezweifeln. Sie leisten derzeit keinen relevanten Beitrag zur Förderung von Bürgertugenden wie Respekt, politische Partizipationsbereitschaft, Gemeinsinn und auch Transparenz, das wichtigste Mittel zur Bekämpfung von Misswirtschaft und Korruptionsanfälligkeit, sondern tragen, genau umgekehrt, dazu bei, die Akzeptanzkrise von Politik bei vielen Bürgern noch zu verstärken. Kirchen, die sich dem Gemeinwesen als Moralagenturen empfehlen, bringen sich selbst nur in Misskredit, wenn sie den von ihnen selbst definierten hohen moralischen Ansprüchen ans Politische nicht zu genügen vermögen. Moralagenturen, in denen es viel Misswirtschaft, Verlogenheit, Schweigekartelle und die systematische Verschleierung von Straftatbeständen gibt, machen sich nicht nur selbst unglaubwürdig, sondern tragen zu einem langfristig gefährlichen, Bürgerengagement und Zivilität unterminierenden Zynismus bei, nach dem in der Politik sowieso nur gelogen und betrogen wird. 
Insoweit besteht eine wichtige Aufgabe der Staats- und Gesellschaftswissenschaften in den kommenden Jahren unter anderem darin, endlich die Kirchen und die mit ihnen verbundenen Sozialholdings von Caritas und Diakonie mit Blick auf die Frage zu erforschen, inwieweit sie überhaupt noch Ligaturen bilden und bürgerschaftliche Tugenden zu fördern vermögen, die durchaus als Voraussetzungen demokratischer Staatlichkeit verstanden werden dürfen.

\section{Res publica revisited: Zur Rolle und Funktion der Staatswissen- schaften im 21. Jahrhundert}

Im Ergebnis wird deutlich, dass wir mit unserer Diskussion über das Verhältnis von Staatswissenschaften und Staatspraxis in einer durchaus beeindruckenden Kontinuität stehen. Sie erlaubt „Überschussenergien“ auf ein historisch informiertes Maß abzusenken, trennt das nicht selten „Modische“ vom Wichtigen und ermöglicht einen vergleichenden Blick auf die die Staatswissenschaften konstituierenden Disziplinen. Dabei wird erkennbar, dass die rasante Entwicklung der das öffentliche Handeln beeinflussenden Rahmenbedingungen nicht nur das politische Steuerungspotential, sondern auch den wissenschaftlichen Erkenntnisprozess nachhaltig beeinflusst. Während die Politik große Schwierigkeiten dokumentiert, sich jenseits situativer Anpassungen auch mit mittel- und langfristig erwartbaren Entwicklungen zu beschäftigen, muss für die Rechts-, Wirtschaftsund Sozialwissenschaften ebenfalls ein zunehmend reaktives Verhalten diagnostiziert werden. Dies ist zunächst dann nicht schädlich, wenn sich damit angemessenes Urteil und „Augenmaß“ verbinden und es auch im historischen wie territorialen Vergleich möglich wird, etwa unterschiedliche Problemlösungen miteinander zu konfrontieren. Diese Haltung wird allerdings dann diskussionswürdig, wenn Wissenschaft nur noch nachvollzieht und darüber hinaus eher selbstreferentiell agiert, während der politisch-administrative Prozess ohne kompetente Kritik und Beratung ,von außen“ bleibt. In diesem Spannungsfeld ergeben sich erkennbar neue Handlungsmöglichkeiten für beide Seiten, bis hin zu einer erweiterten Kooperation. Auch diese freilich bleibt voraussetzungsvoll: So sollte der akademische Bereich wissen, dass „Politikberatung“ sich auf die Funktionsbedingungen politischer Prozesse einzulassen hat, will sie nicht nur wohlfeile Empfehlungen abgeben, und muss die Politik akzeptieren, dass wissenschaftlicher Rat meist „unter Unsicherheit“ steht, gerade deshalb aber einbezogen werden sollte, wenn es um neue und veränderte Reaktionen auftretenden Problemstellungen gegenüber geht. Sich dieser wechselseitigen Bezogenheit (und gleichzeitig Begrenzung) bewusst zu sein und daraus Konsequenzen für das je 
eigene Handeln abzuleiten, erscheint einige Mühen wert sein, zumal die Geschwindigkeit von Veränderungsprozessen beide Akteursgruppen zu überfordern droht. Insofern bietet es sich an, Erkenntnis- wie Handlungspotentiale verstärkt aufeinander zu beziehen, gewiss nicht im Sinne einer wechselseitigen „Arrondierung“, aber im Sinne eines „Aufeinanderhörens“, das Austauschprozesse nahe legt. Dass diese wiederum nicht mehr nur nationalstaatlich zu begrenzen sind, sondern immer auch eine regionale, gelegentlich globale Dimension bergen, sollten die im Themenheft vorgelegten Beiträge deutlich gemacht haben. Die ZSE wird den weiteren Diskussionsprozess begleiten. 